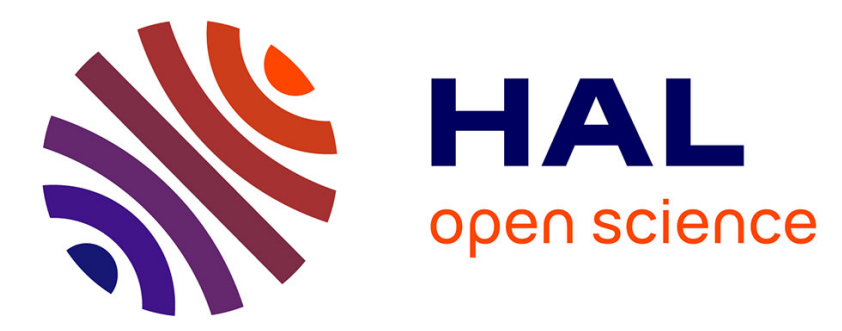

\title{
Multilevel model reduction for uncertainty quantification in computational structural dynamics
}

Olivier Ezvan, Anas Batou, Christian Soize, Laurent Gagliardini

\section{To cite this version:}

Olivier Ezvan, Anas Batou, Christian Soize, Laurent Gagliardini. Multilevel model reduction for uncertainty quantification in computational structural dynamics. Computational Mechanics, 2017, 59 (2), pp.219-246. 10.1007/s00466-016-1348-1 . hal-01391536

\section{HAL Id: hal-01391536 https://hal.science/hal-01391536}

Submitted on 3 Nov 2016

HAL is a multi-disciplinary open access archive for the deposit and dissemination of scientific research documents, whether they are published or not. The documents may come from teaching and research institutions in France or abroad, or from public or private research centers.
L'archive ouverte pluridisciplinaire HAL, est destinée au dépôt et à la diffusion de documents scientifiques de niveau recherche, publiés ou non, émanant des établissements d'enseignement et de recherche français ou étrangers, des laboratoires publics ou privés. 


\section{Multilevel model reduction for uncertainty quantification in computational structural dynamics}

the date of receipt and acceptance should be inserted later

\begin{abstract}
This work deals with an extension of the reduced-order models (ROMs) that are classically constructed by modal analysis in linear structural dynamics of complex structures for which the computational models are assumed to be uncertain. Such an extension is based on a multilevel projection strategy consisting in introducing three reduced-order bases (ROBs) that are obtained by using a spatial filtering methodology of local displacements. This filtering involves global shape functions for the kinetic energy. The proposed multilevel stochastic $\mathrm{ROM}$ is constructed by using the nonparametric probabilistic approach of uncertainties. It allows for affecting a specific level of uncertainties to each type of displacements associated with the corresponding vibration regime, knowing that the local elastic modes are more sensitive to uncertainties than the global elastic modes. The proposed methodology is applied to the computational model of an automobile structure, for which the multilevel stochastic ROM is identified with respect to experimental measurements. This identification is performed by solving a statistical inverse problem.
\end{abstract}

Keywords High modal density - Reduced-Order Model · Uncertainty quantification · Broad frequency band $\cdot$ Structural dynamics

\section{Introduction}

An extension of the classical modal analysis for constructing a reduced-order model (ROM) in computational linear structural dynamics is presented. This ex-

O. Ezvan · A. Batou · C. Soize (凶)

Université Paris-Est, Laboratoire Modélisation et Simulation Multi Echelle, MSME UMR 8208 CNRS, 5 bd Descartes, 77454 Marne-la-Vallee, France

E-mail: christian.soize@univ-paris-est.fr

L. Gagliardini

PSA Peugeot Citroën, Direction Technique et Industrielle, Centre Technique de Vélizy A, Route de Gisy, 78140 Vélizy Villacoublay, France tension is based on a multilevel projection strategy. The computational model is assumed to be uncertain. This paper is a continuation of the work published in [1], for which three new ingredients are presented: (i) the methodology for the construction of the multilevel model reduction is adapted for allowing the probabilistic model of uncertainties to be implemented; (ii) the effective implementation of a multilevel probabilistic model of uncertainties and the statistical inverse problem for its experimental identification; (iii) the experimental validation of the methodology for a very complex computational model of an automobile for which experimental measurements have been performed for 20 manufactured cars of the same type. The complete and detailed developments can be found in [2].

Nowadays, it is well recognized that the predictions in structural dynamics over a broad frequency band by using a computational model, based on the finite element method $[3 ; 4 ; 5]$, must be improved in taking into account the model uncertainties induced by the modeling errors, for which the role increases with the frequency. This means that any model of uncertainties must account for this type of frequency evolution. In addition, it is also admitted that the parametric probabilistic approach of uncertainties is not sufficiently efficient for reproducing the effects of modeling errors. In such a framework, the nonparametric probabilistic approach of uncertainties can be used, but in counter part requires the introduction of a reduced-order model for implementing it. Consequently, these two aspects, frequency-evolution of the uncertainties and reduced-order modeling, lead us to consider the development of a multilevel reduced-order model in computational structural dynamics, which has the capability to adapt the level of uncertainties to each part of the frequency band. This is the purpose of the paper.

In structural dynamics, the low-frequency (LF) band is generally characterized by a low modal density and by frequency response functions (FRFs) exhibiting isolated 
resonances. These are due to the presence of long-wavelength displacements, which are global (the concept of global displacement will be clarified later). In contrast, the high-frequency (HF) band is characterized by a high modal density and by rather smooth FRFs, these being due to the presence of numerous short-wavelength displacements. The intermediate band, the medium-frequency (MF) band, presents a non-uniform modal density and FRFs with overlapping resonances [6]. For the LF band, modal analysis $[7 ; 8 ; 9 ; 10 ; 11 ; 12 ; 13 ; 14]$ is a well-known effective and efficient method, which usually provides a small-dimension ROM whose reduced-order basis (ROB) is constituted of the first elastic modes (i.e. the first structural vibration modes). Energy methods, such as statistical energy analysis $[15 ; 16 ; 17 ; 18 ; 19 ; 20 ; 21 ; 22]$, are commonly used for the $\mathrm{HF}$ band analysis. Various methods have been proposed for analyzing the MF band. A part of these methods are related to deterministic solvers devoted to the classical deterministic linear dynamical equations $[6 ; 23 ; 24 ; 25 ; 11 ; 26 ; 27 ; 28 ; 29 ; 30$; $31 ; 32]$. A second part are devoted to stochastic linear dynamical equations that have been developed for taking into account the uncertainties in the computational models in the MF band (which plays an important role in this band), see for instance $[33 ; 34 ; 35 ; 36 ; 37 ; 38 ; 39 ; 40]$.

In this paper, we are interested in the dynamical analysis of complex structures over a broad frequency band. By complex structure is intended a structure with complex geometry, constituted of heterogeneous materials and more specifically, characterized by the presence of several structural levels, for instance, a structure that is made up of a stiff main part embedding various flexible sub-parts. For such structures, it is possible having, in addition to the usual global-displacements elastic modes associated with their stiff skeleton, the apparition of numerous local elastic modes, which correspond to predominant vibrations of the flexible sub-parts. For such complex structures, which can be encountered for instance in aeronautics, aerospace, automotive (see for instance $[41 ; 42 ; 43]$ ), or nuclear industries, two main difficulties arise from the presence of the local displacements. First, the modal density can be very high as soon the lowfrequency band, yielding a high-dimension ROM when the modal analysis is used (several thousands of elastic modes can be required for such a low-frequency band). Second, such ROMs may suffer from a lack of robustness with respect to uncertainty, because of the presence of the numerous local displacements, which are known to be very sensitive to uncertainties. It should be noted that, for such a complex structure, the engineering objectives may consist in the prediction of the global displacements only, that is to say on predicting the FRFs of observation points belonging to the stiff parts.

There is not much research devoted to the dynamic analysis of structures characterized by the presence of nu- merous local elastic modes intertwined with the global elastic modes. In the framework of experimental modal analysis, techniques for the spatial filtering of the short wavelengths have been proposed [44], based on regularization schemes [45]. In the framework of computational models, the Guyan condensation technique [46], based on the introduction of master nodes at which the inertia is concentrated, allows for the filtering of local displacements. The selection of the master nodes is not obvious for complex structures [47]. Filtering schemes based on the lumped mass matrix approximations have been proposed [48;49;50], but the filtering depends on the mesh and cannot be adjusted. In [16] a basis of global displacements is constructed using a coarse mesh of a finite element model, which, generally, gives big errors for the elastic energy. In order to extract the long-wavelength free elastic modes of a master structure, the free-interface substructuring method has been used [32]. Other computational methods include image processing [51] for identifying the global elastic modes, the global displacements as eigenvectors of the frequency mobility matrix [52], or the extrapolation of the dynamical response using a few elastic modes [53]. In the framework of slender dynamical structures, which exhibit a high modal density in the LF band, simplified equivalent models [54; 55] using beams and plates, or homogenization [56] have been proposed. Using these approaches, the construction of a simplified model is not automatic, requires an expertise, and a validation procedure remains necessary. In addition, these approximations are only valid for the LF band.

For a complex structure for which the elastic modes may not be either purely global elastic modes or purely local elastic modes, the increase of the dimension of the ROM, which is constructed by using the classical modal analysis, can be troublesome. The methodology that would consist in sorting the elastic modes according to whether they be global or local cannot be used because the elastic modes are combinations of both global displacements and local displacements.

Another solution would consist in using substructuring techniques for which reviews can be found in $[57 ; 58 ; 59]$ and for which a state of the art has recently been done in [60]. A brief summary is given hereinafter. The concept of substructures was first introduced by Argyris and Kelsey in 1959 [61] and by Przemieniecki in 1963 [62] and was extended by Guyan and Irons $[46 ; 63]$. Hurty $[64 ; 65]$ considered the case of two substructures coupled through a geometrical interface. Finally, Craig and Bampton [66] adapted the Hurty method. Improvements have been proposed with many variants $[67 ; 68 ; 69 ; 70 ; 71]$, in particular for complex dynamical systems with many appendages considered as substructures (such as a disk with blades) Benfield and Hruda [72]. Another type of methods has been introduced in order to use the structural modes with free geometrical interface for two cou- 
pled substructures instead of the structural modes with fixed geometrical interface (elastic modes) as used in the Craig and Bampton method and as proposed by MacNeal [73] and Rubin [74]. The Lagrange multipliers have also been used to write the coupling on the geometrical interface $[75 ; 76 ; 77 ; 78]$.

The substructuring techniques would require to discard the component modes associated with flexible sub-parts, hence removing their associated local displacements. Unfortunately, for the complex structures considered, there is no clear separation between the skeleton and the substructures for which the displacements would be local. For instance, with fixed thickness, the curvatures of a shell induce stiffened zones with respect to the rigidity of the flat zones. Consequently, in addition to the various embedded equipments within the structure, the complex geometry of the structure is responsible for the fact that there can be no separation of the several structural levels, but rather a continuous series of structural levels. In such conditions, the notion of local displacement is relative. It should be noted that in contrast to the usual long-wavelength global displacements of the LF band, the local displacements associated with the structural sub-levels, which can also appear in the LF band, are characterized by short wavelengths, similarly to HF displacements. As a result, for the complex structures considered, there is an overlap of the three vibration regimes, LF, MF, and HF.

Concerning the taking into account of uncertainties in the computational model, the probabilistic framework is well adapted to the construction of the stochastic models, to the stochastic solvers, and to solve the associated statistical inverse problems for the identification of the stochastic models (for the finite dimension and for the infinite dimension). Hereinafter, we present a brief background that is limited to the probabilistic framework for uncertainty quantification. Several probabilistic approaches can be used depending on the sources of uncertainties in the computational model (model-parameter uncertainties, model uncertainties induced by modeling errors, and variabilities in the real dynamical system).

(i) Output-predictive error method. Several methods are currently available for analyzing model uncertainties. The most popular one is the standard output-predictive error method introduced in [79]. This method has a major drawback because it does not enable the ROM to learn from data.

(ii) Parametric probabilistic methods for model-parameter uncertainties. An alternative family of methods for analyzing model uncertainties is the family of parametric probabilistic methods for the uncertainty quantification. This approach is relatively well developed for model-parameter uncertainties, at least for a reasonably small number of parameters. It consists in constructing prior and posterior stochastic models of uncertain model parameters pertaining, for example, to geometry, to boundary conditions, to material properties, etc $[80 ; 81 ; 82 ; 83 ; 84 ; 85 ; 86 ; 87 ; 88 ; 89 ; 90 ; 91 ; 92 ; 93 ; 94]$. This approach was shown to be computationally efficient for both the computational model and its associated ROM (for example, see [95; 96]), and for large-scale statistical inverse problems $[97 ; 98 ; 99 ; 100 ; 101 ; 102]$. However, it does not take into account neither the model uncertainties induced by modeling errors introduced during the construction of the computational model, nor those due to model reduction.

(iii) Nonparametric probabilistic approach for modeling uncertainties. For modeling uncertainties due to modeling errors, a nonparametric probabilistic approach was introduced in [103], in the context of linear structural dynamics. The methodology is made up of two steps. For the first one, a linear ROM of dimension $n$ is constructed by using the linear computational model with $m$ degrees of freedom (DOFs) and a ROB of dimension $n$. For the second step, a linear stochastic ROM is constructed by substituting the deterministic matrices underlying the linear ROM with random matrices for which the probability distributions are constructed using the Maximum Entropy (MaxEnt) principle [104; 105]. The construction of the linear stochastic ROM is carried out under the constraints generated from the available information such as some algebraic properties (positiveness, integrability of the inverse, etc.) and some statistical information (for example, the equality between mean and nominal values). This nonparametric probabilistic approach has been extended for different ensembles of random matrices and for linear boundary value problems [106; 107]. It was also experimentally validated and applied for linear problems in composites [108], viscoelasticity [109], dynamic substructuring [110; 111], vibroacoustics $[41 ; 40]$, robust design and optimization [112], etc. More recently, the nonparametric approach has been extended to take into account some nonlinear geometrical effects in structural analysis $[113 ; 114]$, but it does not hold for arbitrary nonlinear systems, while the work recently published [115] allows for taking into account any nonlinearity in a ROM.

Recently, a new methodology [116] has been proposed for constructing a stochastic ROM devoted to dynamical structures having numerous local elastic modes in the low-frequency range. The stochastic ROM is obtained by implementing the nonparametric probabilistic approach of uncertainties within a novel ROM whose ROB is constituted of two families, one of global displacements and another one of local displacements. These families are obtained through the introduction, for the kinetic energy, of a projection operator associated with a subspace of piecewise constant functions. The spatial dimension of the subdomains, in which the projected displacements are constant, and which constitute a partition of the domain of the structure, allows for controlling the sepa- 
ration between the global displacements and the local displacements. These subdomains can be seen as macroelements, within which, using such an approximation, no local displacement is permitted. It should be noted that the generation of a domain partition for which the generated subdomains have a similar size (that we call uniform domain partition), necessary for obtaining a spatially uniform filtering criterion, is not trivial for complex geometries. Based on the Fast Marching Method $[117 ; 118]$, a general method has been developed in order to perform the uniform domain partition for a complex finite element mesh, and then implemented for the case of automobile structures [42]. In later work [1;2], the filtering methodology has been generalized through the introduction of a computational framework for the use of any arbitrary approximation subspace for the kinetic energy, in place of the piecewise constant approximation. In particular, polynomial shape functions (with support the whole domain of the structure) have been used for constructing a global-displacements ROM for an automobile. This generalization allows for carrying out an efficient convergence of the global-displacements $\mathrm{ROM}$ with respect to the so-defined filtering (in contrast, constructing several uniform domain partitions of different characteristic sizes can be, in practice, very timeconsuming). In addition, a multilevel ROM has been introduced. The ROB of this ROM is constituted of several families of displacements, which correspond to the several structural levels of the complex structure. More precisely, a multilevel ROM has been presented for which the ROB is constituted of three families, namely the LF, MF-, and HF-type displacements. Each family is obtained by performing a spatial filterings of local displacements. It should be noted that multilevel substructuring techniques can be found in the literature $[119 ; 120 ; 121]$, whose purpose is to accelerate the solution of large-scale generalized eigenvalue problems.

In the present paper, latter filtering methodology (involving polynomial shape functions) is reused, but we present a novel formulation for the construction of a multilevel ROM, which allows for implementing a probabilistic model of uncertainties that is adapted to each vibration regime. This way, the amount of statistical fluctuations for the LF-, MF-, and HF-type displacements can be controlled using the multilevel stochastic ROM that is obtained.

The objective of this paper is double. The first one is to provide a multilevel stochastic ROM that is able to take into account the heterogeneous variability introduced by the overlap of the three vibration regimes. The second one is to provide a predictive ROM whose dimension is smaller than the dimension of the classical ROM constructed by using modal analysis. Both these objectives are to be fulfilled by means of efficient methods that are non-intrusive with respect to commercial software.
The paper is organized as follows. In Section 2, the reference computational model is introduced and the classical construction of the ROM is performed by using modal analysis. Based on this deterministic ROM, the classical stochastic ROM is then constructed by using the nonparametric probabilistic approach of uncertainties. In Section 3, the methodology devoted to the spatial filtering of the global and of the local displacements is presented. These developments are used in Section 4 for defining the multilevel ROM. In this section, the numerical procedure is also detailed and the construction of the multilevel stochastic ROM is given. Finally, in Section 5, the proposed methodology is applied to an automobile, for which the multilevel stochastic ROM is identified by using experimental measurements. The results are compared to those obtained with the classical stochastic ROM.

\section{Notations}

DOF: degree of freedom.

FEM: finite element model.

FRF: frequency response function.

HF: high frequency.

LF: low frequency.

MF: medium frequency (or mid frequency).

ROB: reduced-order vector basis.

ROM: reduced-order model.

C-NROM: classical nominal ROM.

C-SROM: classical stochastic ROM.

ML-NROM: multilevel nominal ROM.

ML-SROM: multilevel stochastic ROM.

$\mathbb{C}^{p}$ : Hermitian space of dimension $p$.

$\mathbb{R}^{p}$ : Euclidean space of dimension $p$.

$\mathcal{S}_{c}$ : vector subspace for the classical ROM.

$\mathcal{S}_{g}$ : vector subspace for the global-displacements ROM. $\mathcal{S}_{\ell}$ : vector subspace for the local-displacements ROM.

$\mathcal{S}_{t}$ : vector subspace for the multilevel ROM.

$\mathcal{S}_{\mathcal{H}}$ : vector subspace for the scale- $\mathcal{H}$ ROM.

$\mathcal{S}_{\mathcal{L}}$ : vector subspace for the scale- $\mathcal{L}$ ROM.

$\mathcal{S}_{\mathcal{M}}$ : vector subspace for the scale- $\mathcal{M}$ ROM.

$\mathcal{S}_{\mathcal{R}}$ : vector subspace for the reduced kinematics.

$\mathcal{S}_{\mathcal{L M}}$ : vector subspace for the scale- $\mathcal{L} \mathcal{M}$ ROM.

$d$ : maximum degree of the polynomial approximation. $m$ : dimension of the FEM (number of DOFs).

$n$ : dimension of $\mathcal{S}_{c}$.

$r$ : dimension of $\mathcal{S}_{\mathcal{R}}$.

$n_{g}$ : dimension of $\mathcal{S}_{g}$.

$n_{\ell}$ : dimension of $\mathcal{S}_{\ell}$.

$n_{t}$ : dimension of $\mathcal{S}_{t}$.

$n_{\mathcal{H}}$ : dimension of $\mathcal{S}_{\mathcal{H}}$.

$n_{\mathcal{L}}$ : dimension of $\mathcal{S}_{\mathcal{L}}$.

$n_{\mathcal{M}}$ : dimension of $\mathcal{S}_{\mathcal{M}}$. 
$n_{\mathcal{L M}}:$ dimension of $\mathcal{S}_{\mathcal{L M}}$

$[B]:$ ROB of $\mathcal{S}_{\mathcal{R}}$ such that $[B]^{T}[\mathrm{M}][B]=\left[I_{r}\right]$.

$\left[B^{\ell}\right]$ : ROB of $\mathcal{S}_{\mathcal{R}}$ such that $\left[B^{\ell}\right]^{T}\left[\mathbb{M}^{\ell}\right]\left[B^{\ell}\right]=\left[I_{r}\right]$.

$\left[I_{p}\right]$ : identity matrix of dimension $p$.

$[\mathrm{M}]$ : mass matrix of the FEM.

$\left[\mathbb{M}^{\ell}\right]$ : lumped approximation of $[\mathbb{M}]$.

$[\Phi]:$ ROB of the classical ROM.

$\left[\Phi^{g}\right]$ : global-displacements ROB.

$\left[\Phi^{\ell}\right]$ : local-displacements ROB.

$\left[\Phi^{t}\right]$ : ROB of the scale- $t$ ROM.

$\left[\Phi^{\mathcal{H}}\right]$ : HF-type displacements ROB.

$\left[\Phi^{\mathcal{L}}\right]$ : LF-type displacements ROB.

$\left[\Phi^{\mathcal{M}}\right]:$ MF-type displacements ROB.

$\left[\Phi^{\mathcal{L M}}\right]:$ ROB of the scale- $\mathcal{L} \mathcal{M}$ ROM.

$[\Psi]$ : ROB of the multilevel ROM.

\section{Classical reduced-order model}

In this section, we introduce the reference computational model and we present the very well known modal analysis method as well as the construction of the associated stochastic ROM that is obtained by using the nonparametric probabilistic approach of uncertainties [103]. This way, basic notions that will be reused later are introduced. In addition, the multilevel stochastic ROM proposed in this paper will be compared to classical stochastic ROM.

\subsection{Reference computational model}

The vibration analysis is performed over a broad frequency band - denoted as $\mathcal{B}$ - by using the finite element method. Let $m$ denote the dimension (number of DOFs) of the finite element model. For all angular frequency $\omega$ belonging to $\mathcal{B}=\left[\omega_{\min }, \omega_{\max }\right]$, the $m$-dimensional complex vector $\mathbb{U}(\omega)$ of displacements is the solution of the matrix equation,

$$
\left(-\omega^{2}[\mathbb{M}]+i \omega[\mathbb{D}]+[\mathbb{K}]\right) \mathbb{U}(\omega)=\mathbb{F}(\omega),
$$

in which $\mathbb{F}(\omega)$ is the $m$-dimensional complex vector of the external forces and where, assuming the structure is fixed on a part of its boundary, $[\mathbb{M}],[\mathbb{D}]$, and $[\mathbb{K}]$ are the positive-definite symmetric $(m \times m)$ real mass, damping, and stiffness matrices.

\subsection{Classical nominal reduced-order model}

For all $\alpha=1, \ldots, m$ the elastic modes $\varphi_{\alpha}$ with associated eigenvalues $\lambda_{\alpha}$ are the solutions of the generalized eigenvalue problem,

$[\mathbb{K}] \boldsymbol{\varphi}_{\alpha}=\lambda_{\alpha}[\mathbb{M}] \boldsymbol{\varphi}_{\alpha}$.
The first $n$ eigenvalues verify $0<\lambda_{1} \leq \lambda_{2} \leq \ldots \leq$ $\lambda_{n}<+\infty$ and the normalization that is chosen for the eigenvectors is such that

$[\Phi]^{T}[\mathbb{M}][\Phi]=\left[I_{n}\right]$,

in which $[\Phi]=\left[\boldsymbol{\varphi}_{1} \ldots \boldsymbol{\varphi}_{n}\right]$, and where $\left[I_{n}\right]$ is the identity matrix of dimension $n$. Such a normalization with the unit generalized mass is always adopted in this paper in which several generalized eigenvalue problems are introduced. In practice, only the first $n$ elastic modes with $n \ll m$ (associated with the lowest eigenvalues or the lowest eigenfrequencies $f_{\alpha}=\sqrt{\lambda_{\alpha}} / 2 \pi$ in $\mathrm{Hz}$ ) are calculated. The $(m \times n)$ real matrix $[\Phi]$ is the $\mathrm{ROB}$ of the classical nominal reduced-order model (C-NROM). The vector subspace spanned by the ROB of the C-NROM is denoted by $\mathcal{S}_{c}$. Using the C-NROM, displacements vector $\mathbb{U}(\omega)$ belong to $\mathcal{S}_{c}$ and we have

$\mathbb{U}(\omega) \simeq[\Phi] \mathfrak{q}(\omega)=\sum_{\alpha=1}^{n} \mathfrak{q}_{\alpha}(\omega) \boldsymbol{\varphi}_{\alpha}$,

where the $n$-dimensional complex vector of generalized coordinates $\mathfrak{q}(\omega)=\left(\mathfrak{q}_{1}(\omega) \ldots \mathfrak{q}_{n}(\omega)\right)$ is the solution of the reduced-matrix equation,

$\left(-\omega^{2}[\mathcal{M}]+i \omega[\mathcal{D}]+[\mathcal{K}]\right) \mathfrak{q}(\omega)=\mathfrak{f}(\omega)$,

in which $\mathfrak{f}(\omega)=[\Phi]^{T} \mathbb{F}(\omega),[\mathcal{D}]=[\Phi]^{T}[\mathbb{D}][\Phi]$ is, in general, a full matrix and where diagonal matrices $[\mathcal{K}]$ and $[\mathcal{M}]$ are such that

$[\mathcal{K}]=[\Phi]^{T}[\mathbb{K}][\Phi]=[\Lambda], \quad[\mathcal{M}]=[\Phi]^{T}[\mathbb{M}][\Phi]=\left[I_{n}\right]$,

in which $[\Lambda]$ is the matrix of the first $n$ eigenvalues.

\subsection{Classical stochastic reduced-order model}

The classical stochastic reduced-order model (C-SROM) is constructed by using the nonparametric probabilistic approach of uncertainties [103] within the C-NROM. In this nonparametric approach, each nominal reduced matrix of dimension $n$, say $[\mathcal{A}](=[\mathcal{M}],[\mathcal{D}]$, or $[\mathcal{K}])$, is replaced by a random matrix, $[\mathcal{A}](=[\mathcal{M}],[\mathcal{D}]$, or $[\mathcal{K}])$ for which the probability distribution has been constructed by using the maximum entropy principle [104; 105] under the following constraints:

- Matrix $[\mathcal{A}]$ is with values in the set of all the positivedefinite symmetric $(n \times n)$ real matrices.

- $E\{[\mathcal{A}]\}=[\mathcal{A}]$, with $E$ the mathematical expectation, which means that the mean matrix is chosen as the nominal matrix.

- $E\left\{\left\|[\mathcal{A}]^{-1}\right\|_{F}^{2}\right\}<+\infty$ for insuring the existence of a second-order solution of the stochastic ROM where $\|.\|_{F}$ is the Frobenius norm.

Introducing the Cholesky factorization $\left[L_{\mathcal{A}}\right]^{T}\left[L_{\mathcal{A}}\right]$ of matrix $[\mathcal{A}]$ in which $\left[L_{\mathcal{A}}\right]$ is an upper-triangular matrix, the construction of random matrix $[\mathcal{A}]$ is given by

$[\mathcal{A}]=\left[L_{\mathcal{A}}\right]^{T}\left[\mathbf{G}_{n}\left(\delta_{\mathcal{A}}\right)\right]\left[L_{\mathcal{A}}\right]$ 
where the random matrix $\left[\mathbf{G}_{n}\left(\delta_{\mathcal{A}}\right)\right]$ (see [103]) is positivedefinite almost surely, for which its mean value is $\left[I_{n}\right]$ and which is parameterized by a dispersion parameter $\delta_{\mathcal{A}}$ defined by

$\delta_{\mathcal{A}}^{2}=\frac{1}{n} E\left\{\left\|\left[\mathbf{G}_{n}\left(\delta_{\mathcal{A}}\right)\right]-\left[I_{n}\right]\right\|_{F}^{2}\right\}$.

The hyperparameter $\delta_{\mathcal{A}}$ of random matrix $\left[\mathbf{G}_{n}\left(\delta_{\mathcal{A}}\right)\right]$ has to verify $0<\delta<\delta_{\max }$ with $\delta_{\max }$ given by

$\delta_{\max }=\sqrt{\frac{n+1}{n+5}}$.

Using the Monte-Carlo simulation method [122], the CSROM allows for computing the random displacements vector $\mathbf{U}(\omega)$ associated with $\mathbb{U}(\omega)$,

$\mathbf{U}(\omega)=[\Phi] \mathcal{Q}(\omega)$,

in which the random complex vector $\mathcal{Q}(\omega)$ of the generalized coordinates is obtained by solving the random matrix equation,

$\left(-\omega^{2}[\mathcal{M}]+i \omega[\mathcal{D}]+[\mathcal{K}]\right) \mathcal{Q}(\omega)=\mathfrak{f}(\omega)$.

The classical ROM is built upon the use of the elastic modes that are present in the frequency band of analysis, $\mathcal{B}$ (or a frequency band slightly wider). In this manner, the dimension of the computational model is reduced while its accuracy is preserved for this band. For the complex structures under consideration, numerous local displacements are intertwined with the global displacements. As a result, among the elastic modes present in $\mathcal{B}$, many have little contribution to the robust dynamical response of the stiff skeleton of the structure that is provided by the C-SROM. Consequently, we present the construction of an adapted ROM that is based on a $\mathrm{ROB}$ in which local displacements have been filtered.

\section{Global-displacements reduced-order model}

In this section, the construction of a new ROM is presented. It is based on the use of a global-displacements ROB instead of the classical ROB made up of elastic modes because the classical ROB can include numerous local elastic modes. In Section 3.1, we present the construction of an unusual mass matrix that is associated with a reduced kinematics for the kinetic energy. In Section 3.2, we use this mass matrix for obtaining unusual eigenvectors that constitute the global-displacements ROB (this unusual mass matrix is not used as the mass matrix for computing the response of the dynamical system). In section 3.3, an efficient and nonintrusive algorithm is proposed for implementing the ROB. Finally, in Section 3.4 , we give the construction of a ROB that is constituted of the complementary local displacements that are neglected in the global-displacements ROM.

\subsection{Reduced kinematics for the kinetic energy}

In order to filter local displacements, a reduced kinematics is introduced for the mass matrix. This reduced kinematics is intended to be such that the local displacements cannot be represented. Instead of using the classical local shape functions of the finite elements for the construction of the mass matrix, we propose the use of $r$ global shape functions, which span a vector subspace denoted by $\mathcal{S}_{\mathcal{R}}$ and which constitute the columns of a $(m \times r)$ real matrix $[B]$. These global shape functions are polynomials for which the maximum degree is denoted by $d$ and that are defined on the whole domain of the structure. The construction of $[B]$ is given in $[1 ; 2]$ and the dimension $r$ of the reduced kinematics is written as

$r=(d+1)(d+2)(d+3) / 2$.

The value of degree $d$ will allow for controlling the filtering of the global displacements and of the local displacements. In order to determine an appropriate value of $d$, a convergence analysis will be carried out with respect to both the deterministic and the stochastic quantities of interest. In the present paper, the strategy proposed consists in approximating the kinetic energy while keeping the elastic energy exact. Such a construction is detailed in $[1 ; 2])$. The mass matrix corresponding to this reduced kinematics will be called the reduced-kinematics mass matrix.

First, the kinetic energy $E_{k}(\mathbb{V}(t))$ associated with any time-dependent real velocity vector $\mathbb{V}(t)$ of dimension $m$ is given by

$E_{k}(\mathbb{V}(t))=\frac{1}{2} \mathbb{V}(t)^{T}[\mathbb{M}] \mathbb{V}(t)$.

Let $\mathbb{V}^{r}(t)$ be the orthogonal projection of $\mathbb{V}(t)$ onto subspace $\mathcal{S}_{\mathcal{R}}$ with respect to the inner-product defined by matrix $[\mathbb{M}]$. Assuming the columns of $[B]$ to be orthonormalized with respect to $[\mathbb{M}]$, the projector $[\mathbb{P}]$ that is such that $\mathbb{V}^{r}(t)=[\mathbb{P}] \mathbb{V}(t)$ is written as

$[\mathbb{P}]=[B][B]^{T}[\mathbb{M}]$.

The rank of the $(m \times m)$ real matrix $[\mathbb{P}]$ is equal to $r \leq m$. The reduced kinetic energy $E_{k}^{r}(\mathbb{V}(t))=E_{k}\left(\mathbb{V}^{r}(t)\right)$ is then written as

$E_{k}^{r}(\mathbb{V}(t))=\frac{1}{2} \mathbb{V}(t)^{T}\left[\mathbb{M}^{r}\right] \mathbb{V}(t)$,

in which the $m$-dimensional reduced-kinematics mass matrix $\left[\mathbb{M}^{r}\right]=[\mathbb{P}]^{T}[\mathbb{M}][\mathbb{P}]$ is positive semidefinite with a rank equal to $r$ and can be written as

$\left[\mathbb{M}^{r}\right]=[\mathbb{M}][B][B]^{T}[\mathbb{M}]$

\subsection{Global-displacements reduced-order basis}

In order to span the global-displacements space denoted by $\mathcal{S}_{g}$ (and which is a subspace of $\mathbb{R}^{m}$ ), mass matrix $[\mathbb{M}]$ 
is replaced by $\left[\mathbb{M}^{r}\right]$ and yields the following generalized eigenvalue problem (that differs from the one used for computing the elastic modes and that cannot be used for computing them),

$[\mathbb{K}] \boldsymbol{\psi}_{\alpha}^{g}=\sigma_{\alpha}^{g}\left[\mathbb{M}^{r}\right] \boldsymbol{\psi}_{\alpha}^{g}$,

in which the eigenvectors $\boldsymbol{\psi}_{\alpha}^{g}$ consist of global displacements and where $\sigma_{\alpha}^{g}$ are the associated eigenvalues. The first $r$ eigenvalues are such that $0<\sigma_{1}^{g} \leq \sigma_{2}^{g} \leq \ldots \leq$ $\sigma_{r}^{g}<+\infty$ and the eigenvalues of rank greater than $r$ are all infinite. Their associated eigenvectors are orthogonal to vector subspace $\mathcal{S}_{\mathcal{R}}$.

It should be noted that (i) the $r$ eigenvectors $\boldsymbol{\psi}_{\alpha}^{g}$ are not orthogonal with respect to mass matrix $[\mathbb{M}]$ and (ii) they will be used for the projection of the computational model defined by Eq.(1), which involves mass matrix [M] and not $\left[\mathbb{M}^{r}\right]$. Let us introduce the $(m \times \nu)$ real matrix, $\left[\Psi^{g}\right]=\left[\boldsymbol{\psi}_{1}^{g} \ldots \boldsymbol{\psi}_{\nu}^{g}\right]$, in which $\nu$ is a given truncation order such that

$\nu \leq r$.

The global-displacements ROB is defined by the first eigenvectors of the dynamical system, which are constrained to belong to the vector space spanned by the $\nu$ columns of the $(m \times \nu)$ real matrix $\left[\Psi^{g}\right]$. The globaldisplacements ROB is denoted by $\left[\Phi^{g}\right]$ whose columns $\varphi_{\alpha}^{g}$ are written as

$\varphi_{\alpha}^{g}=\left[\Psi^{g}\right] \mathbf{r}_{\alpha}$,

in which $\mathbf{r}_{\alpha}$ are the eigenvectors of the small-dimension generalized eigenvalue problem,

$\left(\left[\Psi^{g}\right]^{T}[\mathbb{K}]\left[\Psi^{g}\right]\right) \mathbf{r}_{\alpha}=\lambda_{\alpha}^{g}\left(\left[\Psi^{g}\right]^{T}[\mathbb{M}]\left[\Psi^{g}\right]\right) \mathbf{r}_{\alpha}$.

Introducing the matrix $[R]=\left[\mathbf{r}_{1} \ldots \mathbf{r}_{n_{g}}\right]$, in which $n_{g}$ is a given truncation order that will be defined after, matrix $\left[\Phi^{g}\right]$ can be written as

$\left[\Phi^{g}\right]=\left[\Psi^{g}\right][R]$.

Using Eq. (20) yields

$\left[\Phi^{g}\right]^{T}[\mathbb{K}]\left[\Phi^{g}\right]=\left[\Lambda^{g}\right], \quad\left[\Phi^{g}\right]^{T}[\mathbb{M}]\left[\Phi^{g}\right]=\left[I_{n_{g}}\right]$.

The eigenvalues verify $0<\lambda_{1}^{g} \leq \lambda_{2}^{g} \leq \ldots \leq \lambda_{\nu}^{g}<$ $+\infty$. Only the first $n_{g}$ eigenvectors (associated with the lowest frequencies $f_{\alpha}^{g}=\sqrt{\lambda_{\alpha}^{g}} / 2 \pi$ ) are kept for defining $\left[\Phi^{g}\right]=\left[\boldsymbol{\varphi}_{1}^{g} \ldots \boldsymbol{\varphi}_{n_{g}}^{g}\right]$. The dimension $n_{g}$ of the globaldisplacements subspace $\mathcal{S}_{g}$ is deduced from a cutoff frequency, $f^{c}$, for which $n_{g}$ verifies

$f_{n_{g}}^{g} \leq f^{c}$.

In addition, $n_{g}$ satisfies the inequality,

$n_{g} \leq \nu$.

Cutting frequency $f^{c}$ is a data that must be chosen greater or equal to the upper bound $\omega_{\max } / 2 \pi$ of frequency band $\mathcal{B}$ and that must be adjusted through the analysis of the FRFs. It should be noted that truncation order $\nu$ cannot directly be deduced from the value $\sqrt{\sigma_{\nu}^{g}} / 2 \pi$ because the eigenvalues $\sigma_{\alpha}^{g}$ are not the eigenfrequencies of the dynamical system. For $\nu \leq r$, the following inequality can be proved,

$\lambda_{\nu}^{g} \leq \sigma_{\nu}^{g}$,

for which the difference between $\lambda_{\nu}^{g}$ and $\sigma_{\nu}^{g}$ can be significant.

For brevity, no notation is introduced for the equations related to the global-displacements ROM, which would be similar to Eqs. (4) and (5) and which, anyway, would not be used.

3.3 Numerical implementation for the construction of the global-displacements ROB

As the mass matrix $\left[\mathbb{M}^{r}\right]$ is a full $(m \times m)$ matrix, this matrix is not assembled. In addition, the eigenvalue problem defined by Eq. (17) requires the knowledge of matrices $[\mathbb{M}]$ and $[\mathbb{K}]$, which can involve problems for the commercial software. The purpose of this section is to present an efficient method for the construction of the globaldisplacements ROB.

Let $\left[\mathbb{M}^{\ell}\right]$ be the diagonal matrix that is a lumped approximation of mass matrix [M]. For avoiding the use of $[\mathbb{M}]$, the following approximation of $\left[\mathbb{M}^{r}\right]$ is introduced,

$\left[\mathbb{M}^{r}\right] \simeq\left[\mathbb{M}^{\ell}\right]\left[B^{\ell}\right]\left[B^{\ell}\right]^{T}\left[\mathbb{M}^{\ell}\right]$

in which the construction of $\left[B^{\ell}\right]$ is similar to the one of $[B]$ (see Section 3.1 ) but is such that $\left[B^{\ell}\right]^{T}\left[\mathbb{M}^{\ell}\right]\left[B^{\ell}\right]=$ $\left[I_{r}\right]$. The projector $[\mathbb{P}]$ defined by Eq. (14) is then approximated by

$[\mathbb{P}] \simeq\left[B^{\ell}\right]\left[B^{\ell}\right]^{T}\left[\mathbb{M}^{\ell}\right]$.

Equation (26) consists in applying the reduced kinematics (associated with a given maximum degree for the polynomial approximation) to a diagonal lumped approximation $\left[\mathbb{M}^{\ell}\right]$ of consistent mass matrix $[\mathbb{M}]$, instead of applying it to $[\mathbb{M}]$. Since a diagonal approximation can be obtained by a kinematic reduction for which the displacement field is constant over each finite element, the error induced by Eq. (26) is related to the one obtained by approximating the polynomial shape functions by functions that are a constant in each finite element. For a polynomial with a low degree, the spatial variation is slow and, as it is assumed that the FEM is constructed upon a fine mesh, such an approximation can therefore be considered negligible. Nevertheless, in this paper, high-degree polynomial shape functions have been used. In order to estimate the maximum effect of such an approximation given by Eq. (26) (for the case of highdegree polynomial shape functions), a prior analysis has been carried out, consisting in computing deterministic 
quantities of interest (such as moduli of FRFs in logscale) by two different global-displacements ROMs, one with the lumped mass matrix and the other one with the consistent matrix, and in comparing the results given by the two ROMs, which have shown that the error is effectively negligible.

A double projection method is presented, which allows the explicit use of matrix $[\mathbb{K}]$. It consists in projecting Eq. (17) onto subspace $\mathcal{S}_{c}$ that is associated with the classical ROB made up of the elastic modes. This subspace is supposed to provide, upon the use of a sufficiently large value of the number $n$ of elastic modes, an accurate representation on frequency band $\mathcal{B}$. Then, without loss of model fidelity, such a projection can be obtained by writing

$\mathcal{S}_{g} \subseteq \mathcal{S}_{c}$

that is satisfied if $\boldsymbol{\psi}_{\alpha}^{g}$ is written as

$\boldsymbol{\psi}_{\alpha}^{g}=[\Phi] \mathbf{s}_{\alpha}$,

in which $\mathbf{s}_{\alpha}$ is a $n$-dimensional real vector that has to be calculated as follows. By using Eq. (29), the projection of Eq. (17) yields the following reduced-dimension generalized eigenvalue problem,

$\left([\Phi]^{T}[\mathbb{K}][\Phi]\right) \mathbf{s}_{\alpha}=\sigma_{\alpha}^{g}\left([\Phi]^{T}\left[\mathbb{M}^{r}\right][\Phi]\right) \mathbf{s}_{\alpha}$.

The matrix $[\Phi]^{T}[\mathbb{K}][\Phi]$ is the diagonal matrix $[\Lambda]$ defined in Eq. (6), which is available. By using Eq. (26), the full matrix $[\Phi]^{T}\left[\mathbb{M}^{r}\right][\Phi]$ can be computed as

$[\Phi]^{T}\left[\mathbb{M}^{r}\right][\Phi] \simeq[N][N]^{T}$,

in which the $(n \times r)$ real matrix $[N]$ that is defined by

$[N]=[\Phi]^{T}\left[\mathbb{M}^{\ell}\right]\left[B^{\ell}\right]$

is also available. Introducing $[S]=\left[\mathbf{s}_{1} \ldots \mathbf{s}_{\nu}\right]$ and using Eq. (29), matrix $\left[\Psi^{g}\right]$ can be rewritten as

$\left[\Psi^{g}\right]=[\Phi][S]$.

Denoting as $\left[\Sigma^{g}\right]$ the diagonal matrix of the first $\nu$ eigenvalues $\sigma_{\alpha}^{g}$ and recalling the choice of a unit generalized mass normalization, it can then be deduced that the reduced matrices involved in Eq. (20) are such that

$\left[\Psi^{g}\right]^{T}[\mathbb{K}]\left[\Psi^{g}\right]=\left[\Sigma^{g}\right]$ and $\left[\Psi^{g}\right]^{T}[\mathbb{M}]\left[\Psi^{g}\right]=[S]^{T}[S]$.

Remark 1. Physical interpretation of the filtering strategy. Introducing the $(m \times n)$ real matrix $\left[\Phi^{r}\right]=[\mathbb{P}][\Phi]$, we obtain $[\Phi]^{T}\left[\mathbb{M}^{r}\right][\Phi]=\left[\Phi^{r}\right]^{T}[\mathbb{M}]\left[\Phi^{r}\right]$. The following interpretation of Eq. (30) can be given. While reduced stiffness matrix $[\Phi]^{T}[\mathbb{K}][\Phi]$ is the projection of stiffness matrix $[\mathbb{K}]$ onto the basis $[\Phi]$ of the elastic modes (including both the global and the local displacements), the reduced mass matrix $\left[\Phi^{r}\right]^{T}[\mathbb{M}]\left[\Phi^{r}\right]$ is the projection of mass matrix $[\mathbb{M}]$ onto displacements represented by matrix $\left[\Phi^{r}\right]$, which belong to subspace $\mathcal{S}_{\mathcal{R}}$ and in which some local displacements are filtered.

Remark 2. Computational efficiency. It should be noted that the numerical rank, $R$, of $[N][N]^{T}$ is such that $R \leq$ $r$ and $R \leq n$. Similarly to Eq. (18), truncation order $\nu$ must satisfy the inequality $\nu \leq R$. For solving the generalized eigenvalue problem defined by Eq. (30), three cases are considered.

- For $r<n$, a thin SVD (see [123]) of the $(n \times r)$ real matrix $[\Lambda]^{-1 / 2}[N]$ is performed for a lower cost.

- For $r \gg n, R=n$ is verified and matrix $[N][N]^{T}$ is positive definite. For this case, the usual algorithms are used.

- For the intermediate case for which $R$ is close to $n$, the SVD approach is more efficient in order to obtain a good accuracy.

\subsection{Local-displacements reduced-order basis}

In the rest of the paper, it is assumed that the above numerical implementation of the global-displacements ROB is used. In this section, we present the construction of a local-displacements ROB. The vector subspace associated with the local-displacements ROB, denoted by $\mathcal{S}_{\ell}$, is the orthogonal complement of subspace $\mathcal{S}_{g}$ of $\mathcal{S}_{c}$ with respect to the inner-product defined by matrix $[\mathbb{M}]$. In particular, $\mathcal{S}_{c}$ is the orthogonal direct sum of $\mathcal{S}_{g}$ with $\mathcal{S}_{\ell}$,

$\mathcal{S}_{c}=\mathcal{S}_{g} \oplus \mathcal{S}_{\ell}$

Thanks to this definition of $\mathcal{S}_{\ell}$, its ROB, denoted by $\left[\Phi^{\ell}\right]$, satisfies the orthogonality condition

$\left[\Phi^{g}\right]^{T}[\mathbb{M}]\left[\Phi^{\ell}\right]=[0]$

as well as the following equality

$\left[\Phi^{\ell}\right]=[\Phi]\left[Q^{\ell}\right]$

in which $\left[Q^{\ell}\right]$ is the $\left(n \times n_{\ell}\right)$ real matrix of the coordinates in the basis defined by $[\Phi]$, and where the dimension $n_{\ell}$ is such that

$n_{\ell}=n-n_{g}$.

Let $\left[Q^{g}\right]$ be the $\left(n \times n_{g}\right)$ real matrix such that

$\left[Q^{g}\right]=[S][R]$

Using Eqs. (21), (33), and (39) yield

$\left[\Phi^{g}\right]=[\Phi]\left[Q^{g}\right]$.

From Eqs. (3), (36), (37), and (40), the following orthogonality property can be deduced,

$\left[Q^{g}\right]^{T}\left[Q^{\ell}\right]=[0]$.

Let $[Z]$ be the $\left(n \times n_{\ell}\right)$ real matrix whose columns are the right-singular vectors associated with the $n_{\ell}$ zero singular values of the SVD of $\left[Q^{g}\right]^{T}$ (and which, consequently, 
is an algebraic basis of the nullspace of $\left.\left[Q^{g}\right]^{T}\right)$. By construction, matrix $[Z]$ verifies

$[Z]^{T}[Z]=\left[I_{n_{\ell}}\right]$.

Equation (41) is satisfied for $\left[Q^{\ell}\right]$ expressed as

$\left[Q^{\ell}\right]=[Z][U]$,

in which $[U]$ is a $\left(n_{\ell} \times n_{\ell}\right)$ real matrix of coordinates in the basis defined by $[Z]$. The local-displacements ROB represented by matrix $\left[\Phi^{\ell}\right]$ is then defined by the first eigenvectors of the dynamical system, which are constrained to belong to the vector space spanned by the $n_{\ell}$ columns of the $\left(m \times n_{\ell}\right)$ real matrix $[\Phi][Z]$. The columns $\varphi_{\alpha}^{\ell}$ of $\left[\Phi^{\ell}\right]$ are thus written as

$\varphi_{\alpha}^{\ell}=[\Phi][Z] \mathbf{u}_{\alpha}$,

in which, thanks to Eqs.(6) and (42), it can be deduced that the columns $\mathbf{u}_{\alpha}$ of $[U]$ are the eigenvectors of the following standard eigenvalue problem,

$\left([Z]^{T}[\Lambda][Z]\right) \mathbf{u}_{\alpha}=\lambda_{\alpha}^{\ell} \mathbf{u}_{\alpha}$.

In this Section 3, a general method has been presented for obtaining a global-displacements ROM, for which the construction of the associated ROB depends on the choice of parameters $d$ (maximum degree of the polynomial approximation), $\nu$ (truncation order), and $f^{c}$ (cutoff frequency). The dimension $n_{g}$ of the ROM results from the values of these parameters. Suitable values of $d$ and $\nu$ must be tuned in order to obtain a smaller dimension $n_{g} \leq n$ while preserving the fidelity of the computational model. As higher frequencies are characterized by vibrations of shorter wavelength, the choice of the values for $d$ and $\nu$ strongly depend on the value of frequency $f^{c}$. The construction of a ROB made up of local displacements, which is complementary to the global-displacements ROB, has also been proposed and will be useful for the construction of a multilevel ROM.

\section{Multilevel reduced-order model}

\subsection{Formulation of the multilevel ROM}

In this section, the previous developments are used in the construction of a multilevel ROM, for which three ROBs associated with the low-, medium-, and high-frequency bands (LF, MF, HF) are introduced. In contrast to the $\mathrm{HF}$ band, the LF band is associated with long-wavelength global displacements, while the MF band is a combination of global and local displacements with more or less short wavelength. For the complex structures considered, there is an overlap of the three vibration regimes. For instance, numerous local elastic modes can be found in the low-frequency band. The previously introduced filtering strategy is therefore used in order to separate the LF-, MF-, and HF-type displacements. The filtering methodology presented in Sections 3.3 and 3.4 can be represented by the following mapping,

$\mathcal{F}_{1}:\left(\mathcal{S}_{c} ; d, \nu, f^{c}\right) \longmapsto\left(\mathcal{S}_{g}, \mathcal{S}_{\ell}\right)$,

which will be used for defining the multilevel ROM. As the classical ROM, the multilevel ROM is devoted to the vibration analysis over whole band $\mathcal{B}$, which can be decomposed into the three following bands,

$\mathcal{B}=\mathcal{B}_{\mathcal{L}} \cup \mathcal{B}_{\mathcal{M}} \cup \mathcal{B}_{\mathcal{H}}$

We now present the basic ideas concerning the construction of the multilevel ROM based on the introduction of three successive filterings, which are defined through mapping $\mathcal{F}_{1}$. We introduce the cutoff frequency $f_{\mathcal{H}}^{c}$ for which the value has to be chosen by considering the value of the upper bound of $\mathcal{B}_{\mathcal{H}}$. The (global-displacements) vector subspace $\mathcal{S}_{t}$, which includes the totality of the remaining considered displacements and which is associated with the multilevel ROM, is given by

$\left(\mathcal{S}_{t}, \mathcal{S}_{t}^{\perp}\right)=\mathcal{F}_{1}\left(\mathcal{S}_{c} ; d_{\mathcal{H}}, \nu_{\mathcal{H}}, f_{\mathcal{H}}^{c}\right)$,

in which the values of the parameters $d_{\mathcal{H}}$ and $\nu_{\mathcal{H}}$ are tuned in order to obtain a decreased dimension while preserving the fidelity of the ROM up to frequency $f_{\mathcal{H}}^{c}$. It should be recalled that subspace $\mathcal{S}_{c}$ is assumed to be associated with an accurate classical ROM over band $\mathcal{B}$. The (local-displacements) vector subspace $\mathcal{S}_{t}^{\perp}$, verifying $\mathcal{S}_{c}=\mathcal{S}_{t} \oplus \mathcal{S}_{t}^{\perp}$, is not used (the local displacements spanning this subspace are discarded in order to decrease the dimension of the proposed ROM). For carrying out the next filterings, similarly to the double projection method associated with Eq. (28), the computational model is now projected onto subspace $\mathcal{S}_{t}$, which is supposed to be associated with a sufficiently accurate representation.

Similarly, we introduce the cutoff frequency $f_{\mathcal{M}}^{c}$ which is be chosen by considering the value of the upper bound of $\mathcal{B}_{\mathcal{M}}$. The (local-displacements) vector subspace, $\mathcal{S}_{\mathcal{H}}$, associated with the $\mathrm{HF}$ vibration regime is given by

$\left(\mathcal{S}_{\mathcal{L M}}, \mathcal{S}_{\mathcal{H}}\right)=\mathcal{F}_{1}\left(\mathcal{S}_{t} ; d_{\mathcal{M}}, \nu_{\mathcal{M}}, f_{\mathcal{M}}^{c}\right)$

where $\mathcal{S}_{\mathcal{L M}}$ is the complementary (global-displacements) subspace belonging to $\mathcal{S}_{t}$. The values of the parameters $d_{\mathcal{M}}$ and $\nu_{\mathcal{M}}$ are tuned such that the ROB associated with $\mathcal{S}_{\mathcal{L M}}$ yields an adequate representation up to frequency $f_{\mathcal{M}}^{c}$. We have the decomposition $\mathcal{S}_{t}=\mathcal{S}_{\mathcal{L} \mathcal{M}} \oplus \mathcal{S}_{\mathcal{H}}$.

Finally, the cutoff frequency $f_{\mathcal{L}}^{c}$ is introduced, which is chosen by considering the value of the upper bound of $\mathcal{B}_{\mathcal{L}}$. The (global-displacements) vector subspace, $\mathcal{S}_{\mathcal{L}}$, associated with the LF vibration regime, is given by

$\left(\mathcal{S}_{\mathcal{L}}, \mathcal{S}_{\mathcal{M}}\right)=\mathcal{F}_{1}\left(\mathcal{S}_{\mathcal{L} \mathcal{M}} ; d_{\mathcal{L}}, \nu_{\mathcal{L}}, f_{\mathcal{L}}^{c}\right)$

where $\mathcal{S}_{\mathcal{M}}$ is the complementary (local-displacements) subspace belonging to $\mathcal{S}_{\mathcal{L M}}$. The values of the parameters $d_{\mathcal{L}}$ and $\nu_{\mathcal{L}}$ are tuned such that the ROB associated with $\mathcal{S}_{\mathcal{L}}$ and mainly made up of global displacements, yields a sufficiently accurate representation up 
to frequency $f_{\mathcal{L}}^{c}$ It should be noted that, with a similar manner as the previous one, the proposed construction makes the assumption that subspace $\mathcal{S}_{\mathcal{L M}}$ constructed with a higher polynomial degree (that has been tuned for covering a broader) frequency band ( $\mathrm{LF} \cup \mathrm{MF})$ ), is associated with a sufficiently accurate representation (for the LF band). Finally, we have the decomposition $\mathcal{S}_{\mathcal{L M}}=\mathcal{S}_{\mathcal{L}} \oplus \mathcal{S}_{\mathcal{M}}$ and consequently,

$\mathcal{S}_{t}=\mathcal{S}_{\mathcal{L}} \oplus \mathcal{S}_{\mathcal{M}} \oplus \mathcal{S}_{\mathcal{H}}$

\subsection{Implementation of the multilevel nominal ROM}

\subsubsection{Numerical procedure}

In this section, the numerical implementation is detailed. We summarize the steps introduced in Sections 3.3 and 3.4 that are devoted to the construction of the globaldisplacements ROB and of the local-displacements ROB. Similarly to Eq. (46), the procedure is then compacted in a mapping, $\mathcal{F}_{2}$, which allows for defining the algebraic quantities associated with the multilevel ROM formulated in Section 4.1. In the following, the steps for calculating the outputs of mapping $\mathcal{F}_{2}$ are given.

For this, it is assumed that maximum degree $d$ of the polynomial approximation, truncation order $\nu$, and cutoff frequency $f^{c}$ are given and some new notations are introduced in order to generalize the numerical procedure. Let $\left[\Lambda^{0}\right]$ be a diagonal matrix of dimension $n_{0}$ whose diagonal elements are strictly positive. Let $\left[Q^{0}\right]$ be a $\left(n_{1} \times n_{0}\right)$ real matrix for which $n_{1} \geq n_{0}$ and such that $\left[Q^{0}\right]^{T}\left[Q^{0}\right]=\left[I_{n_{0}}\right]$. Let $\left[N^{0}\right]$ be a $\left(n_{1} \times r_{\max }\right)$ real matrix for which $r_{\max } \geq r$, with $r=(d+1)(d+2)(d+3) / 2$. These three matrices are the input parameters of mapping $\mathcal{F}_{2}$ in addition to the filtering parameters that are $d, \nu$, and $f^{c}$. The outputs of mapping $\mathcal{F}_{2}$ will allow us to define the construction of all the matrices involved in the multilevel ROM. It should be noted that, aside from the multilevel ROM, mapping $\mathcal{F}_{2}$ allows for constructing the global- and the local-displacements ROBs defined in Section 3 by using, as inputs the quantities $\left[\Lambda^{0}\right]=[\Lambda]$, $\left[Q^{0}\right]=\left[I_{n}\right]$ with $n_{1}=n_{0}=n$, and $\left[N^{0}\right]=[N]$.

The matrix $\left[N^{0}\right]$ is associated with a reduced kinematics for which the maximum degree of the polynomials is greater or equal to $d$, and which can already have been used during a previous filtering. Let $\left[N_{r}^{0}\right]$ be the $\left(n_{1} \times r\right)$ matrix constituted of the first $r$ columns of $\left[N^{0}\right]$. In addition, the previous filtering is associated with a change of basis defined by matrix $\left[Q^{0}\right]$. Let $\left[N_{r}\right]$ be the $\left(n_{0} \times r\right)$ real matrix defined by

$\left[N_{r}\right]=\left[Q^{0}\right]^{T}\left[N_{r}^{0}\right]$.

The eigenvectors $\mathbf{s}_{\alpha}$ and the associated eigenvalues $\sigma_{\alpha}^{g}$ are calculated (similarly to Eq. (30)) as

$\left[\Lambda^{0}\right] \mathbf{s}_{\alpha}=\sigma_{\alpha}^{g}\left(\left[N_{r}\right]\left[N_{r}\right]^{T}\right) \mathbf{s}_{\alpha}$.
Introducing the matrix $[S]=\left[\mathbf{s}_{1} \ldots \mathbf{s}_{\nu}\right]$ and the matrix $\left[\Sigma^{g}\right]$ of the eigenvalues $\sigma_{\alpha}^{g}$, the eigenvectors $\mathbf{r}_{\alpha}$ and the associated eigenvalues $\lambda_{\alpha}^{g}$ are calculated (similarly to Eq. (20)) as

$\left[\Sigma^{g}\right] \mathbf{r}_{\alpha}=\lambda_{\alpha}^{g}\left([S]^{T}[S]\right) \mathbf{r}_{\alpha}$.

Dimension $n_{g}$ of the global-displacements ROB is the maximum integer $\alpha$ verifying the inequality $f_{\alpha}^{g} \leq f^{c}$, in which $f_{\alpha}^{g}=\sqrt{\lambda_{\alpha}^{g}} / 2 \pi$. Then, matrix $\left[\Lambda^{g}\right]$ is made up of the $n_{g}$ eigenvalues $\lambda_{\alpha}^{g}$. Let $[R]$ be the matrix such that $[R]=\left[\mathbf{r}_{1} \ldots \mathbf{r}_{n_{g}}\right]$ and let $\left[Q^{g}\right]$ be the matrix defined by $\left[Q^{g}\right]=[S][R]$. Introducing $[C]=\left[Q^{g}\right]^{T}$, the following SVD is performed,

$[C]=\left[U_{C}\right]\left[\Sigma_{C}\right]\left[V_{C}\right]^{T}$.

The columns of $\left[V_{C}\right]$ associated with the $n_{\ell}$ zero singular values in $\left[\Sigma_{C}\right]$ allow for obtaining the $\left(n_{0} \times n_{\ell}\right)$ real matrix $[Z]$ with $n_{\ell}=n_{0}-n_{g}$. Finally, the eigenvectors $\mathbf{u}_{\alpha}$ and the associated eigenvalues $\lambda_{\alpha}^{\ell}$ are calculated (similarly to Eq. (45)) as

$\left([Z]^{T}\left[\Lambda^{0}\right][Z]\right) \mathbf{u}_{\alpha}=\lambda_{\alpha}^{\ell} \mathbf{u}_{\alpha}$,

which allows for obtaining the matrix $[U]=\left[\mathbf{u}_{1} \ldots \mathbf{u}_{n_{\ell}}\right]$ and the diagonal matrix $\left[\Lambda^{\ell}\right]$ of the $n_{\ell}$ eigenvalues $\lambda_{\alpha}^{\ell}$, followed by the construction of matrix $\left[Q^{\ell}\right]=[Z][U]$.

The procedure summarized hereinbefore allows the following mapping to be constructed,

$$
\begin{aligned}
& \left(\left[\Lambda^{g}\right],\left[Q^{g}\right],\left[N_{r}\right],\left[\Lambda^{\ell}\right],\left[Q^{\ell}\right]\right)= \\
& \mathcal{F}_{2}\left(\left[\Lambda^{0}\right],\left[Q^{0}\right],\left[N^{0}\right] ; d, \nu, f^{c}\right),
\end{aligned}
$$

in which the outputs $\left[Q^{g}\right]$ and $\left[Q^{\ell}\right]$ verify the following orthonormality properties,

$$
\begin{aligned}
{\left[Q^{g}\right]^{T}\left[Q^{g}\right]=\left[I_{n_{g}}\right],\left[Q^{\ell}\right]^{T}\left[Q^{\ell}\right]=\left[I_{n_{\ell}}\right] } & \\
& {\left[Q^{g}\right]^{T}\left[Q^{\ell}\right]=[0] . }
\end{aligned}
$$

These properties can be deduced from Eqs. (20), (34), (37), (41), (42), (43), (45), and from the fact that the unit generalized mass normalization is used.

\subsubsection{Construction of the reduced-order bases}

First filtering. In Eq. (48), vector subspace $\mathcal{S}_{t}$ is obtained by the projection onto $\mathcal{S}_{c}$ in which $d_{\mathcal{H}}, \nu_{\mathcal{H}}$, and $f_{\mathcal{H}}^{c}$ are the filtering parameters. Let $n_{t}$ be the dimension of the multilevel ROM and let $r_{\mathcal{H}}=\left(d_{\mathcal{H}}+1\right)\left(d_{\mathcal{H}}+2\right)\left(d_{\mathcal{H}}+3\right) / 2$. The real matrices $\left[\Lambda^{t}\right],\left[Q^{t}\right]$, and $\left[N^{t}\right]$, respectively of dimensions $\left(n_{t} \times n_{t}\right),\left(n \times n_{t}\right)$, and $\left(n \times r_{\mathcal{H}}\right)$ are defined as the first three outputs of mapping $\mathcal{F}_{2}$ such that

$$
\begin{aligned}
\left(\left[\Lambda^{t}\right],\left[Q^{t}\right],\left[N^{t}\right], \sim, \sim\right) & = \\
& \mathcal{F}_{2}\left([\Lambda],\left[I_{n}\right],[N] ; d_{\mathcal{H}}, \nu_{\mathcal{H}}, f_{\mathcal{H}}^{c}\right),
\end{aligned}
$$


in which $[N]=[\Phi]^{T}\left[\mathbb{M}^{\ell}\right]\left[B^{\ell}\right]$. The symbol $\sim$ indicates that the corresponding output variables are not calculated. The ROB $\left[\Phi^{t}\right]$ associated with $\mathcal{S}_{t}$ is given by

$\left[\Phi^{t}\right]=[\Phi]\left[Q^{t}\right]$,

in which, thanks to Eq. (58), $\left[Q^{t}\right]$ verifies

$\left[Q^{t}\right]^{T}\left[Q^{t}\right]=\left[I_{n_{t}}\right]$.

Second filtering. In Eq. (49), vector subspaces $\mathcal{S}_{\mathcal{L M}}$ and $\mathcal{S}_{\mathcal{H}}$ have their ROB defined through the following outputs of mapping $\mathcal{F}_{2}$,

$$
\begin{aligned}
\left(\left[\Lambda^{\mathcal{L M}}\right],\left[Q^{\mathcal{L M}}\right],\left[N^{\mathcal{L M}}\right],\left[\Lambda^{\mathcal{H}}\right],\left[Q^{\mathcal{H}}\right]\right)= \\
\mathcal{F}_{2}\left(\left[\Lambda^{t}\right],\left[Q^{t}\right],\left[N^{t}\right] ; d_{\mathcal{M}}, \nu_{\mathcal{M}}, f_{\mathcal{M}}^{c}\right),
\end{aligned}
$$

with $d_{\mathcal{M}} \leq d_{\mathcal{H}}, \nu_{\mathcal{M}} \leq n_{t}$, and where (similarly to Eq. (58)) the following properties are verified,

$$
\begin{array}{r}
{\left[Q^{\mathcal{L M}}\right]^{T}\left[Q^{\mathcal{L M}}\right]=\left[I_{n_{\mathcal{L} \mathcal{M}}}\right],\left[Q^{\mathcal{H}}\right]^{T}\left[Q^{\mathcal{H}}\right]=\left[I_{n_{\mathcal{H}}}\right],} \\
{\left[Q^{\mathcal{L M}}\right]^{T}\left[Q^{\mathcal{H}}\right]=[0],}
\end{array}
$$

with $n_{\mathcal{L M}}=\operatorname{dim}\left(\mathcal{S}_{\mathcal{L M}}\right)$ and $n_{\mathcal{H}}=\operatorname{dim}\left(\mathcal{S}_{\mathcal{H}}\right)$. The ROBs $\left[\Phi^{\mathcal{L M}}\right]$ and $\left[\Phi^{\mathcal{H}}\right]$ of subspaces $\mathcal{S}_{\mathcal{L} \mathcal{M}}$ and $\mathcal{S}_{\mathcal{H}}$ are given by

$$
\left[\Phi^{\mathcal{L M}}\right]=\left[\Phi^{t}\right]\left[Q^{\mathcal{L M}}\right], \quad\left[\Phi^{\mathcal{H}}\right]=\left[\Phi^{t}\right]\left[Q^{\mathcal{H}}\right]
$$

Third filtering. In Eq. (50), vector subspaces $\mathcal{S}_{\mathcal{L}}$ and $\mathcal{S}_{\mathcal{M}}$ have their ROB defined through the following outputs of mapping $\mathcal{F}_{2}$,

$$
\begin{aligned}
\left(\left[\Lambda^{\mathcal{L}}\right],\left[Q^{\mathcal{L}}\right],\right. & \left.\sim,\left[\Lambda^{\mathcal{M}}\right],\left[Q^{\mathcal{M}}\right]\right)= \\
& \mathcal{F}_{2}\left(\left[\Lambda^{\mathcal{L} \mathcal{M}}\right],\left[Q^{\mathcal{L M}}\right],\left[N^{\mathcal{L} \mathcal{M}}\right] ; d_{\mathcal{L}}, \nu_{\mathcal{L}}, f_{\mathcal{L}}^{c}\right),
\end{aligned}
$$

with $d_{\mathcal{L}} \leq d_{\mathcal{M}}, \nu_{\mathcal{L}} \leq n_{\mathcal{L} \mathcal{M}}$, and where (similarly to Eq. (58)) the following properties are verified,

$$
\begin{aligned}
{\left[Q^{\mathcal{L}}\right]^{T}\left[Q^{\mathcal{L}}\right]=\left[I_{n_{\mathcal{L}}}\right],\left[Q^{\mathcal{M}}\right]^{T}\left[Q^{\mathcal{M}}\right] } & =\left[I_{n_{\mathcal{M}}}\right], \\
& {\left[Q^{\mathcal{L}}\right]^{T}\left[Q^{\mathcal{M}}\right]=[0], }
\end{aligned}
$$

with $n_{\mathcal{L}}=\operatorname{dim}\left(\mathcal{S}_{\mathcal{L}}\right)$ and $n_{\mathcal{M}}=\operatorname{dim}\left(\mathcal{S}_{\mathcal{M}}\right)$. The ROBs $\left[\Phi^{\mathcal{L}}\right]$ and $\left[\Phi^{\mathcal{M}}\right]$ of subspaces $\mathcal{S}_{\mathcal{L}}$ and $\mathcal{S}_{\mathcal{M}}$ are given by $\left[\Phi^{\mathcal{L}}\right]=\left[\Phi^{\mathcal{L} \mathcal{M}}\right]\left[Q^{\mathcal{L}}\right]$ and $\left[\Phi^{\mathcal{M}}\right]=\left[\Phi^{\mathcal{L} \mathcal{M}}\right]\left[Q^{\mathcal{M}}\right]$, which yields

$$
\left[\Phi^{\mathcal{L}}\right]=\left[\Phi^{t}\right]\left[Q^{\mathcal{L M}}\right]\left[Q^{\mathcal{L}}\right], \quad\left[\Phi^{\mathcal{M}}\right]=\left[\Phi^{t}\right]\left[Q^{\mathcal{L} \mathcal{M}}\right]\left[Q^{\mathcal{M}}\right]
$$

\subsubsection{Construction of the reduced-order models}

Scale-S reduced-order model. For $\mathcal{S}$ representing any one of the symbols $t, \mathcal{L} \mathcal{M}, \mathcal{H}, \mathcal{L}$, and $\mathcal{M}$, when using the scale- $\mathcal{S}$ ROM, displacements vector $\mathbb{U}(\omega)$ belong to the subspace $\mathcal{S}_{\mathcal{S}}$ that is defined as the space spanned by the columns of matrix $\left[\Phi^{\mathcal{S}}\right]$, and are written as

$\mathbb{U}(\omega) \simeq\left[\Phi^{\mathcal{S}}\right] \mathfrak{q}^{\mathcal{S}}(\omega)$.
The $n_{\mathcal{S}}$-dimensional complex vector of generalized coordinates $\mathfrak{q}^{\mathcal{S}}(\omega)$ is the solution of the reduced-matrix equation,

$\left(-\omega^{2}\left[\mathcal{M}^{\mathcal{S}}\right]+i \omega\left[\mathcal{D}^{\mathcal{S}}\right]+\left[\mathcal{K}^{\mathcal{S}}\right]\right) \mathfrak{q}^{\mathcal{S}}(\omega)=\mathfrak{f}^{\mathcal{S}}(\omega)$,

in which it can easily be proved that

$$
\begin{aligned}
\mathfrak{f}^{\mathcal{S}}(\omega) & =\left[\Phi^{\mathcal{S}}\right]^{T} \mathbb{F}(\omega), \quad\left[\mathcal{M}^{\mathcal{S}}\right]=\left[\Phi^{\mathcal{S}}\right]^{T}[\mathbb{M}]\left[\Phi^{\mathcal{S}}\right]=\left[I_{n_{\mathcal{S}}}\right] \\
{\left[\mathcal{D}^{\mathcal{S}}\right] } & =\left[\Phi^{\mathcal{S}}\right]^{T}[\mathbb{D}]\left[\Phi^{\mathcal{S}}\right], \quad\left[\mathcal{K}^{\mathcal{S}}\right]=\left[\Phi^{\mathcal{S}}\right]^{T}[\mathbb{K}]\left[\Phi^{\mathcal{S}}\right]=\left[\Lambda^{\mathcal{S}}\right]
\end{aligned}
$$

Multilevel nominal reduced-order model. The $\left(m \times n_{t}\right)$ ROB of the multilevel nominal ROM (ML-NROM) is represented by matrix $[\Psi]$ that is written as

$[\Psi]=\left[\left[\Phi^{\mathcal{L}}\right]\left[\Phi^{\mathcal{M}}\right]\left[\Phi^{\mathcal{H}}\right]\right]$

Using the ML-NROM, displacement vector $\mathbb{U}(\omega)$ is approximated by

$$
\begin{aligned}
\mathbb{U}(\omega) \simeq[\Psi] \mathbf{q}(\omega) \\
=\left[\Phi^{\mathcal{L}}\right] \mathbf{q}^{\mathcal{L}}(\omega)+\left[\Phi^{\mathcal{M}}\right] \mathbf{q}^{\mathcal{M}}(\omega)+\left[\Phi^{\mathcal{H}}\right] \mathbf{q}^{\mathcal{H}}(\omega),
\end{aligned}
$$

in which $\mathbf{q}(\omega)=\left(\mathbf{q}^{\mathcal{L}}(\omega), \mathbf{q}^{\mathcal{M}}(\omega), \mathbf{q}^{\mathcal{H}}(\omega)\right)$ with $\mathbf{q}^{\mathcal{L}}(\omega)$ in $\mathbb{C}^{n_{\mathcal{L}}}, \mathbf{q}^{\mathcal{M}}(\omega)$ in $\mathbb{C}^{n_{\mathcal{M}}}$, and $\mathbf{q}^{\mathcal{H}}(\omega)$ in $\mathbb{C}^{n_{\mathcal{H}}}$. The complex vector $\mathbf{q}(\omega)$ is the solution of the reduced-matrix equation,

$\left(-\omega^{2}[M]+i \omega[D]+[K]\right) \mathbf{q}(\omega)=\mathbf{f}(\omega)$,

in which

$$
\begin{aligned}
\mathbf{f}(\omega)=[\Psi]^{T} \mathbb{F}(\omega), \quad[M]=[\Psi]^{T}[\mathbb{M}][\Psi], \\
{[D]=[\Psi]^{T}[\mathbb{D}][\Psi], \quad[K]=[\Psi]^{T}[\mathbb{K}][\Psi] . }
\end{aligned}
$$

Let us define $\left[W^{\mathcal{L}}\right]=\left[Q^{\mathcal{L M}}\right]\left[Q^{\mathcal{L}}\right],\left[W^{\mathcal{M}}\right]=\left[Q^{\mathcal{L} \mathcal{M}}\right]\left[Q^{\mathcal{M}}\right]$, and $\left[W^{\mathcal{H}}\right]=\left[Q^{\mathcal{H}}\right]$. Let $([A],[\mathbb{A}])$ representing any one of the symbols $([M],[\mathbb{M}]),([D],[\mathbb{D}])$, and $([K],[\mathbb{K}])$. From Eqs. (64), (67), and (71), it can be deduced that a blockwriting of $[A]=[\Psi]^{T}[\mathbb{A}][\Psi]$ can be written as

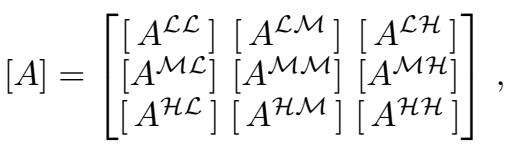

for which the matrix blocks are defined as follows. For $\mathcal{I}$ and $\mathcal{J}$ in $\{\mathcal{L}, \mathcal{M}, \mathcal{H}\}$, the $\left(n_{\mathcal{I}} \times n_{\mathcal{J}}\right)$ real matrix $\left[A^{\mathcal{I} \mathcal{J}}\right]$ is given by

$\left[A^{\mathcal{I} \mathcal{J}}\right]=\left[W^{\mathcal{I}}\right]^{T}\left[\mathcal{A}^{t}\right]\left[W^{\mathcal{J}}\right]$

in which, thanks to Eq. $(60),\left[\mathcal{A}^{t}\right]=\left[Q^{t}\right]^{T}[\mathcal{A}]\left[Q^{t}\right]$ with $[\mathcal{A}]=[\Phi]^{T}[\mathbb{A}][\Phi]$. Moreover, Eqs. (61), (63), and (66) yield

$\left[W^{\mathcal{I}}\right]^{T}\left[W^{\mathcal{I}}\right]=\left[I_{n_{\mathcal{I}}}\right],\left[W^{\mathcal{I}}\right]^{T}\left[W^{\mathcal{J}}\right]=[0]$ if $\mathcal{I} \neq \mathcal{J}$,

from which it can be deduced that $[M]=\left[I_{n_{t}}\right]$. 


\subsection{Multilevel stochastic reduced-order model}

Similarly to the C-SROM, the multilevel stochastic ROM (ML-SROM) is based on the nonparametric probabilistic approach of uncertainties. This approach allows for taking into account both the model-parameter uncertainties and the model uncertainties induced by the modeling errors. The ML-NROM previously presented is based on the use of three orthogonal ROBs represented by the matrices $\left[\Phi^{\mathcal{L}}\right],\left[\Phi^{\mathcal{M}}\right]$, and $\left[\Phi^{\mathcal{H}}\right]$, which are constituted of LF-, MF-, and HF-type displacements, respectively. For instance, as explained in Section 4.1, the LF-type displacements consist of long-wavelength global displacements, in contrast to the short-wavelength local displacements of the HF band. When a small design change is performed in the structure, the local displacements that exist in the modified part of the structure are likely to vary a lot, whereas the shape of the global displacements is not really modified. Subsequently and as it is well known, the local displacements are more sensitive to uncertainties than the global displacements.

For each given random matrix $[\mathbf{A}]$ representing $[\mathbf{M}],[\mathbf{D}]$ or $[\mathbf{K}]$ of the ML-SROM, which is associated with the corresponding deterministic matrix $[A]$ representing $[M]$, $[D]$ or $[K]$ of the ML-NROM, three dispersion hyperparameters, $\delta_{A}^{\mathcal{L}}, \delta_{A}^{\mathcal{M}}$, and $\delta_{A}^{\mathcal{H}}$ are introduced. These hyperparameters are intended to allow each type of displacements to be affected by a particular level of uncertainties. For $\mathcal{S}$ equal to $\mathcal{L}, \mathcal{M}$ or $\mathcal{H}$, the dispersion hyperparameter $\delta_{A}^{\mathcal{S}}$ is such (see Eq. (8)) that

$\left(\delta_{A}^{\mathcal{S}}\right)^{2}=\frac{1}{n_{\mathcal{S}}} E\left\{\left\|\left[\mathbf{G}_{n_{\mathcal{S}}}\left(\delta_{A}^{\mathcal{S}}\right)\right]-\left[I_{n_{\mathcal{S}}}\right]\right\|_{F}^{2}\right\}$.

We define the random matrix $\left[\mathbf{G}_{A}\right]$ with values in the set of all the positive-definite symmetric $\left(n_{t} \times n_{t}\right)$ real matrices, such that

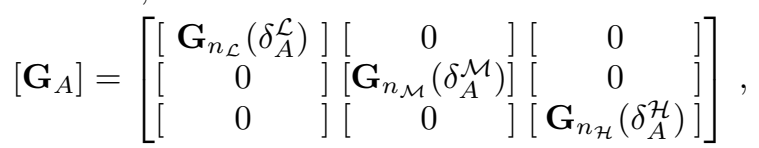

in which the random matrices $\left[\mathbf{G}_{n_{\mathcal{L}}}\left(\delta_{A}^{\mathcal{L}}\right)\right],\left[\mathbf{G}_{n_{\mathcal{M}}}\left(\delta_{A}^{\mathcal{M}}\right)\right]$, and $\left[\mathbf{G}_{n_{\mathcal{H}}}\left(\delta_{A}^{\mathcal{H}}\right)\right]$, with dimensions $\left(n_{\mathcal{L}} \times n_{\mathcal{L}}\right),\left(n_{\mathcal{M}} \times n_{\mathcal{M}}\right)$, and $\left(n_{\mathcal{H}} \times n_{\mathcal{H}}\right)$, are statistically independent and are constructed similarly to the $(n \times n)$ random matrix $[\mathcal{A}]$ defined by Eq. (7). Performing the Cholesky factorization $[A]=\left[L_{A}\right]^{T}\left[L_{A}\right]$, in which $\left[L_{A}\right]$ is an upper-triangular matrix, the random matrix $[\mathbf{A}]$ is constructed as

$[\mathbf{A}]=\left[L_{A}\right]^{T}\left[\mathbf{G}_{A}\right]\left[L_{A}\right]$.

The ML-SROM allows the random displacements vector $\mathbf{U}(\omega)$ associated with $\mathbb{U}(\omega)$ to be obtained as

$\mathbf{U}(\omega)=[\Psi] \mathbf{Q}(\omega)$,

in which the $\mathbb{C}^{n_{t}}$-valued random variable $\mathbf{Q}(\omega)$ is the solution of the random matrix equation,

$\left(-\omega^{2}[\mathbf{M}]+i \omega[\mathbf{D}]+[\mathbf{K}]\right) \mathbf{Q}(\omega)=\mathbf{f}(\omega)$.

For all $\omega$ in $\mathcal{B}$, the random equation defined by Eq. (82) is solved with the Monte-Carlo simulation method.
4.4 Comments about the construction and the identification of the ML-SROM

A multilevel ROM has been presented for which the construction depends on filtering parameters. Three filterings are successively performed. For each filtering, the filtering parameters have to be chosen such that the approximation is satisfactory for the considered frequency band. The satisfaction criterion can be based either on deterministic or on stochastic quantities of interest. In Section 4.3, a multilevel stochastic ROM has been introduced, for which the uncertainty is controlled by three dispersion hyperparameters associated with each one of the three families, namely, LF-, MF-, and HF-type displacements, which constitute the ROB of the ROM. In the framework of the nonparametric probabilistic approach of uncertainties (which uses a random matrix theory), the dispersion hyperparameters allow for controlling the level of statistical fluctuations for each reduced matrix of the ROM (around its mean matrix) . Thus, the hyperparameters take into account uncertainty in a global manner for each type of forces (the inertial forces, the damping forces, and the elastic forces). The statistical inverse identification (or calibration) of the hyperparameters of the ML-SROM depends on the filtering parameters, which has been made for constructing the ML-NROM. For example, one set of filtering parameters can lead us to a low-dimension ROM with low eigenvalue density while another set of filtering parameters can lead us to a high-dimension ROM with high eigenvalue density. With fixed dispersion hyperparameters, the stochastic ROM with a high dimension is likely to exhibit a higher level of uncertainty than the ROM with a low dimension.

\section{Application to an automobile structure}

\subsection{Problem definition}

\subsubsection{Experimental measurements: excitation force, observation points, and frequency band of analysis}

Experimental measurements of some FRFs have been carried out for $n_{e}=20$ nominally identical cars over a broad frequency band, $\mathcal{B}=2 \pi \times[10,900] \mathrm{Hz}$. For each car, the same excitation force is applied to one of the engine fasteners and the acceleration (following a given direction) is measured at two locations referenced as observation 1 that is far away from the excitation force and as observation 2 that is close to the excitation force.

\subsubsection{Computational model}

The finite element model is very dense and complex, including several kinds of elements (springs, bars, beams, 
plates, shells, volume elements), rigid bodies, and constraint equations. The total number of DOFs is $m=$ $7,872,583$. A view of the finite element model is displayed in Fig. 1.

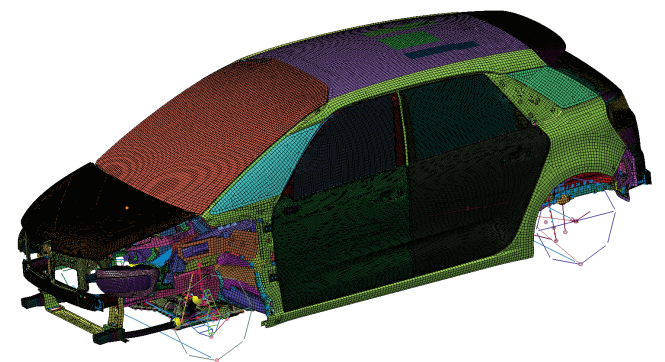

Fig. 1 View of the finite element model of the automobile.

\subsubsection{Modal density characterizing the dynamics and} definition of the $L F, M F$, and $H F$ bands

An intensive computational effort has been carried out for calculating the 24,578 elastic modes that are in the frequency band $[0,2200] \mathrm{Hz}$. The graph of the modal density corresponding to these 24,578 elastic modes is displayed in Fig. 2. From this calculation, it can be seen

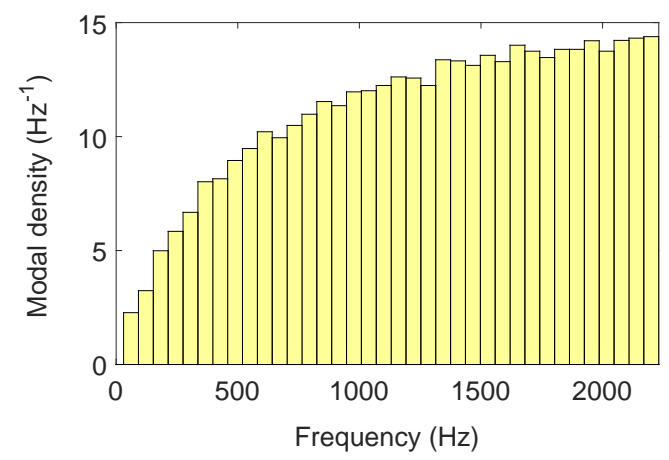

Fig. 2 Modal density calculated with the computational model.

that there are 7,258 elastic modes in frequency band $\mathcal{B}$. There are several possible definitions of the frequency bands $\mathcal{B}_{\mathcal{L}}, \mathcal{B}_{\mathcal{M}}$, and $\mathcal{B}_{\mathcal{H}}$ for a complex dynamical system. The definitions greatly depend on the use that is made of the bands defined. In the present framework devoted to the construction of a multilevel model reduction in structural vibrations, we choose the approach proposed in [40], which is based on the analysis of the graph of the unwrapped phase as a function of the frequency in logarithmic scale for observation 1 that is far away from the excitation force and consequently, for which the propagation follows a long path. It is recalled that the unwrapped phase corrects the radian phase angles by adding multiples of $\pm 2 \pi$ when absolute jumps between two consecutive sampled frequencies are greater than or equal to the jump tolerance of $\pi$ radians. It is known that in the LF range, the phase rotates of $\pi$ around an eigenfrequency while, in the HF band, the unwrapped phase decreases quasilinearly. Figure 3 displays the graph of the unwrapped phase obtained with the computational model, which is compared to the 20 graphs that correspond to the experimental measurements. The analysis of this figure allows for defining the following frequency bands $\left.\left.\mathcal{B}_{\mathcal{L}}=[10,70] \mathrm{Hz}, \mathcal{B}_{\mathcal{M}}=\right] 70,300\right] \mathrm{Hz}$, and $\left.\left.\mathcal{B}_{\mathcal{H}}=\right] 300,900\right]$ $\mathrm{Hz}$. There are 149 elastic modes in low-frequency band $\mathcal{B}_{\mathcal{L}}, 1,202$ elastic modes in medium-frequency band $\mathcal{B}_{\mathcal{M}}$, and 5,897 elastic modes in high-frequency band $\mathcal{B}_{\mathcal{H}}$, or, in average, about 2.5 modes per $\mathrm{Hz}$ in $\mathcal{B}_{\mathcal{L}}, 5$ modes per $\mathrm{Hz}$ in $\mathcal{B}_{\mathcal{M}}$, and 10 modes per $\mathrm{Hz}$ in $\mathcal{B}_{\mathcal{H}}$. A modal density of 2.5 modes per $\mathrm{Hz}$ is quite high for the LF band of such a structure. This unusual feature is due to the presence of numerous local displacements in addition to the usual global displacements. For higher frequencies, the density of local elastic modes keeps increasing, which yields a large number of elastic modes for the modal analysis.

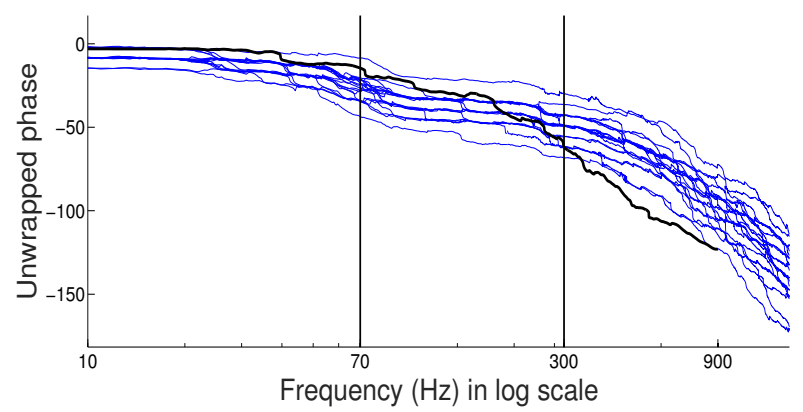

Fig. 3 Graph of the unwrapped phase as a function of the frequency in logarithmic scale for observation 1. Computational model (thick line) and 20 experimental measurements (thin lines).

\subsubsection{Damping model for the automobile}

The damping matrix $[\mathbb{D}]$ does not correspond to the finite element discretization of physical damping and dissipation phenomena. The damping model is introduced at the ROM level. For each ROM, the damping matrix is constructed by using a modal damping model. In the LF band and in the MF band, a multi-parameter modal damping model is fitted by using the experimental FRFs. In the HF band, a one-parameter modal damping model is identified by using the experimental FRFs, for which the parameter is denoted by $c_{\mathcal{H}}$ (for the details of such a construction, see [2]). For the deterministic ROMs, three 
cases have to be considered in order to properly define the damping model, depending on which ROM is used.

- The reduced damping matrix of the C-NROM involved in Eq. (5) is defined by $[\mathcal{D}]=2\left[\Xi\left(c_{\mathcal{H}}\right)\right][\Lambda]^{1 / 2}$. The diagonal matrix $[\Lambda]$ is defined by Eq. (6) and $\left[\Xi\left(c_{\mathcal{H}}\right)\right]$ is a $(n \times n)$ diagonal matrix of modal damping rates that depend on parameter $c_{\mathcal{H}}$, which has to be identified with respect to the experimental measurements, in a deterministic framework. In such a case, Eq. (5) consists of a diagonal matrix equation.

- The reduced damping matrix of the scale-S ROM involved in Eq. (69) is defined by the equation $\left[\mathcal{D}^{\mathcal{S}}\right]=$ $2\left[\Xi^{\mathcal{S}}\left(c_{\mathcal{H}}\right)\right]\left[\Lambda^{\mathcal{S}}\right]^{1 / 2}$. The diagonal matrix $\left[\Lambda^{\mathcal{S}}\right]$ is defined by Eq. $(70)$ and $\left[\Xi^{\mathcal{S}}\left(c_{\mathcal{H}}\right)\right]$ is a $\left(n_{\mathcal{S}} \times n_{\mathcal{S}}\right)$ diagonal matrix of damping rates, which depend on parameter $c_{\mathcal{H}}$ that has to be identified with respect to the experimental measurements, in a deterministic framework. In such a case, Eq. (69) consists of a diagonal matrix equation.

- Concerning the ML-NROM, reduced stiffness matrix $[K]$ involved in Eq. (73) is a full matrix. In order to solve latter matrix equation, one possibility is to perform a change of basis in order to diagonalize the reduced matrices $[K]$ and $[M]$. Doing so leads us back to scale- $t$ ROM, for which the definition of the damping matrix $\left[\mathcal{D}^{t}\right]$ is given in the item just before.

For the stochastic ROMs, two cases have to be considered in order to properly define the random matrix of the damping model, depending on which stochastic ROM is used.

- For constructing the random reduced damping matrix $[\mathcal{D}]$ of the C-SROM involved in Eq. (11), Eq. (7) is not used. In order to solve Eq. (11), the random generalized eigenvalue problem associated with random reduced matrices $[\mathcal{K}]$ and $[\mathcal{M}]$ is solved, which yields the diagonal matrix, $[\boldsymbol{\Lambda}]$, of the random eigenvalues and the matrix, $[\boldsymbol{\Phi}]$, of the associated random eigenvectors. Equation (11) is then projected onto the stochastic ROB $[\boldsymbol{\Phi}]$. In order to obtain a diagonal matrix equation, random damping matrix $[\mathcal{D}]$ of the $\mathrm{C}$ SROM is then constructed similarly to deterministic reduced damping matrix $[\mathcal{D}]$ of the $\mathrm{C}-\mathrm{NROM}$ given hereinbefore, except that diagonal matrix $[\Lambda]$ is replaced by $[\boldsymbol{\Lambda}]$. The parameter $c_{\mathcal{H}}$ has to be identified with respect to the experimental measurements, in a stochastic framework.

- In Eq. (80), the definition given to the random damping matrix $[\mathbf{D}]$ of the ML-SROM involved in Eq. (82) is not used. In order to solve Eq. (82), the random generalized eigenvalue problem associated with random reduced matrices $[\mathbf{K}]$ and $[\mathbf{M}]$ is solved, which yields the diagonal matrix, $[\boldsymbol{\Sigma}]$, of the random eigenvalues and the matrix, $[\boldsymbol{\Psi}]$, of the associated random eigenvectors. Equation (82) is then projected onto the stochastic ROB $[\boldsymbol{\Psi}]$. In order to obtain a diagonal matrix equation, the random reduced damping matrix
[D] of the ML-SROM is then constructed similarly to the deterministic reduced damping matrix $[D]$ of the ML-NROM given just before, except that diagonal matrix $\left[\Lambda^{t}\right]$ is replaced by $[\boldsymbol{\Sigma}]$. The parameter $c_{\mathcal{H}}$ has to be identified with respect to the experimental measurements, in a stochastic framework.

\subsubsection{Definition of the observations}

Let $\mathbb{U}_{(1)}(\omega), \ldots, \mathbb{U}_{\left(n_{j}\right)}(\omega)$ be the $n_{j}$ scalar observations that are made up of DOFs or combinations of DOFs of the displacements vector $\mathbb{U}(\omega)$. In this application, we have $n_{j}=2$ (corresponding to the two experimental observations). For each $j$, the computed observation is defined as the modulus $\omega \mapsto u_{j}^{c}(\omega)$ in $\mathrm{dB}$ scale,

$u_{j}^{c}(\omega)=20 \log _{10}\left|\mathbb{U}_{(j)}(\omega)\right|$,

in which $|$.$| is the modulus of a complex number. The$ counterpart for the experimental measurements of the $k=1, \ldots, n_{e}$ cars (with $n_{e}=20$ ) is denoted by $\omega \mapsto$ $u_{j, k}^{e}(\omega)$ for which the definition is the same as the definition of $u_{j}^{c}(\omega)$.

\subsubsection{Defining the objective function used for the convergence analyses of the deterministic computational} ROMs

In order to define a distance between the computed FRFs and the experimental FRFs, an objective function, $J_{d}$, is defined by

$J_{d}^{2}=\frac{1}{n_{j}} \sum_{j=1}^{n_{j}} \frac{1}{n_{e}} \sum_{k=1}^{n_{e}} \frac{1}{|\mathcal{B}|} \int_{\mathcal{B}}\left(u_{j}^{c}(\omega)-u_{j, k}^{e}(\omega)\right)^{2} d \omega$,

with $|\mathcal{B}|=\omega_{\max }-\omega_{\min }$. It should be noted that the construction of $J_{d}$ would remain unchanged if, in Eq. (84), the displacements were replaced by their corresponding accelerations.

\subsubsection{Defining the objective function used for the identification of the stochastic computational ROMs}

The filtering parameters and the hyperparameters of the stochastic computational ROMs have to be identified with respect to the experimental measurements (solving a statistical inverse problem). Let $U_{j}^{e}(\omega)$ be the realvalued random variable for which $u_{j, 1}^{e}(\omega), \ldots, u_{j, n_{e}}^{e}(\omega)$ are $n_{e}$ independent realizations. Let $U_{j}^{c}(\omega)$ be the realvalued random variable corresponding to the deterministic quantity $u_{j}^{c}(\omega)$. The following objective function, $J_{s}$, is introduced

$J_{s}=\frac{1}{n_{j}} \sum_{j=1}^{n_{j}} J_{s, j}$ 
in which the objective function $J_{s, j}$ associated with observation $j=1, \ldots, n_{j}$ is written as

$J_{s, j}=\frac{1}{|\mathcal{B}|} \int_{\mathcal{B}} \operatorname{OVL}\left(U_{j}^{c}(\omega), U_{j}^{e}(\omega)\right) d \omega$.

In Eq. (86), the function $(X, Y) \mapsto \operatorname{OVL}(X, Y)$ is defined by

$\operatorname{OVL}(X, Y)=1-\frac{1}{2} \int_{\mathbb{R}}\left|p_{X}(x)-p_{Y}(x)\right| d x$,

in which $X$ and $Y$ are real-valued random variables, for which $p_{X}$ and $p_{Y}$ are the probability density functions. Function OVL is known as the overlapping coefficient [124]. For all $j=1, \ldots, n_{j}$ and all $\omega$ in $\mathcal{B}, n_{\text {sim }}$ realizations of random variable $U_{j}^{c}(\omega)$ are computed by using the Monte-Carlo simulation method. The probability density functions are estimated by using the kernel density estimation method. It can easily be proved that the values of $J_{s}$, of $J_{s, j}$ for $j=1, \ldots, n_{j}$, and of the OVL function, are between 0 and 1 (with 1 meaning a perfect match).

5.2 Classical nominal ROM and classical stochastic $\mathrm{ROM}$

In a first step, the dimension $n$ of the C-NROM (deterministic) is calculated by performing a convergence analysis of $J_{d}$ as a function of $n$. For this value of $n$, the $\mathrm{C}-\mathrm{NROM}$ is used for computing the deterministic FRFs that are compared to their experimental counterparts. In a second step, for the value of $n$ determined in the first step, the hyperparameters of the C-SROM (stochastic) are identified by maximizing the objective function $J_{s}$. Using the identified values of the hyperparameters, the C-SROM is used for estimating the confidence regions of the random FRFs, which are compared to the experimental measurements.

\subsubsection{First step: $C$-NROM}

Convergence analysis of $J_{d}$ as a function of $n$. The CNROM is obtained by using Eqs. (4) and (5). A convergence analysis of the C-NROM is performed with respect to its dimension $n$ and to parameter $c_{\mathcal{H}}$ that controls the damping model. For each value of $n$, the optimal value of $c_{\mathcal{H}}$ is identified, in minimizing $J_{d}$. For each pair $\left(n, c_{\mathcal{H}}(n)\right)$, Fig. 4 displays the graph of $J_{d}$ as a function of $n$ and shows that convergence is reached starting from $n=7,000$. Nevertheless, we choose $n=8,450$ (for which $f_{n}=1,000 \mathrm{~Hz}$ ), in order (1) to have subspace $\mathcal{S}_{c}$ sufficiently rich for the construction of subspace $\mathcal{S}_{t} \subseteq \mathcal{S}_{c}$ of the multilevel ROM and (2) to have a classical ROM associated with eigenfrequencies covering more than whole band $\mathcal{B}$ for obtaining a satisfying stochastic ROM.

Deterministic FRFs and experimental comparisons. The

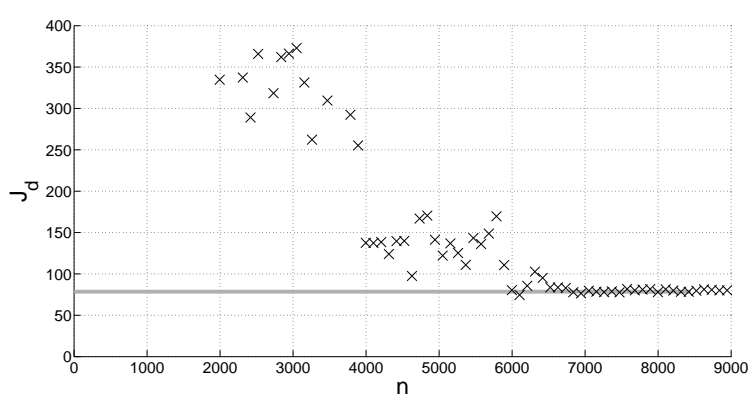

Fig. 4 Convergence of the C-NROM with respect to its dimension: value of $J_{d}$ for several values of $n$ (black crosses). Horizontal light-gray line: value of $J_{d}$ for $n=8,450$.

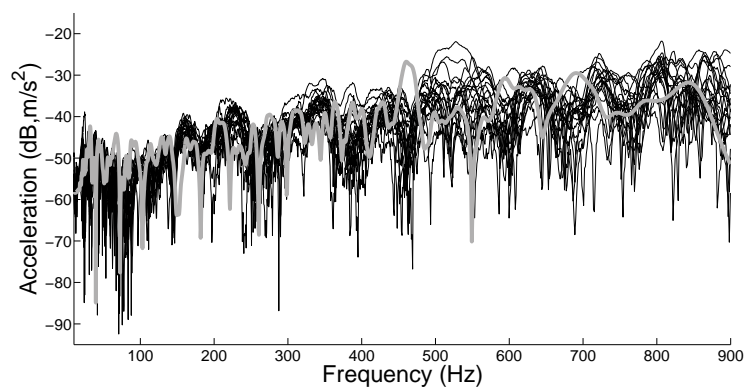

Fig. 5 Observation 1: experimental FRF measurements (black lines), deterministic FRF using the C-NROM (gray line)

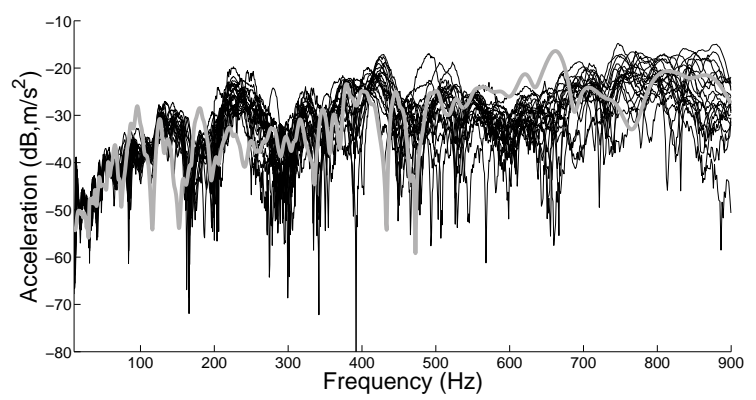

Fig. 6 Observation 2: experimental FRF measurements (black lines), deterministic FRF using the C-NROM (gray line)

FRFs provided by the C-NROM, associated with observation 1 (acceleration related to the displacement $\omega \mapsto$ $u_{1}^{c}(\omega)$ ) and with observation 2 (acceleration related to the displacement $\left.\omega \mapsto u_{2}^{c}(\omega)\right)$, are plotted in Figs. 5 and 6 and are compared to the $n_{e}=20$ experimental FRFs (accelerations related to displacements $\omega \mapsto u_{j, k}^{e}(\omega)$ with $\left.k=1, \ldots, n_{e}\right)$. These figures clearly show that

- the experimental variabilities strongly increase with the frequency (it should be noted that the relative important experimental variabilities in the LF band for observation 1 that can be seen in Fig. 5 is due to measurement noise). 
- significant differences between the prediction of the computational model and the experimental measurements can be observed, in particular in the MF band.

- the big experimental variabilities cannot be represented by a deterministic computational model, but require the prediction of confidence regions with a stochastic computational model.

\subsubsection{Second step: $C-S R O M$}

Experimental identification of the $C$-SROM. The C-SROM

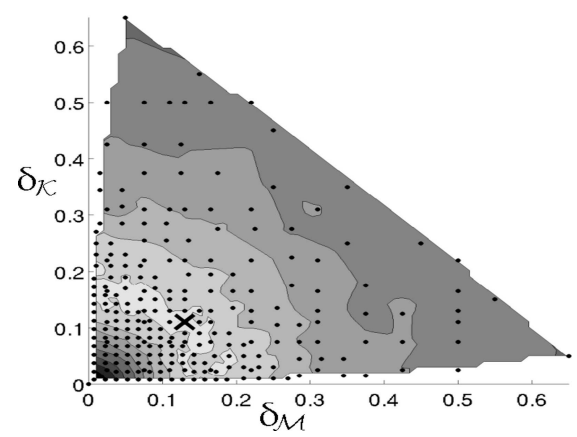

Fig. 7 Plot of function $\left(\delta_{\mathcal{M}}, \delta_{\mathcal{K}}\right) \mapsto J_{s}\left(\delta_{\mathcal{M}}, \delta_{\mathcal{K}}\right)$ for the identification of hyperparameters $\delta_{\mathcal{M}}$ and $\delta_{\mathcal{K}}$ of the C-SROM. Black dots: sampling points, black cross: optimal point.

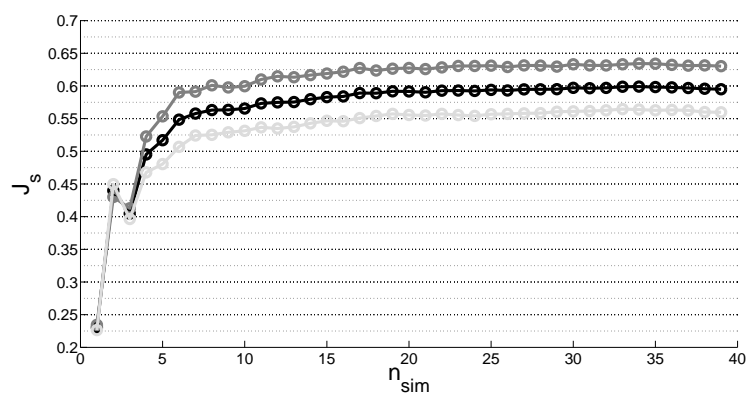

Fig. 8 Convergence of objective function $J_{s}$ (black line), of $J_{s, 1}$ (dark-gray line), and of $J_{s, 2}$ (light-gray line) with respect to number of simulations $n_{\mathrm{sim}}$.

is obtained by using Eqs. (10) and (11) for which the dispersion hyperparameters and damping parameter $c_{\mathcal{H}}$ must be identified with respect to the experimental measurements, by solving a statistical inverse problem. The random damping matrix $[\mathcal{D}]$ of the $\mathrm{C}-\mathrm{SROM}$ is the one defined in Section 5.1.4 and consequently, there is no dispersion hyperparameter $\delta_{\mathcal{D}}$. The statistical inverse problem consists in computing the optimal values $\delta_{\mathcal{M}}^{\mathrm{opt}}, \delta_{\mathcal{K}}^{\mathrm{opt}}$, and $c_{\mathcal{H}}^{\text {opt }}$ of the optimization problem defined as the maximization of the objective function $J_{s}$ with respect to the three hyperparameters $\delta_{\mathcal{M}}, \delta_{\mathcal{K}}$, and $c_{\mathcal{H}}$ in the set of their admissible values. The optimization problem is not convex and a trial method is used by introducing a fine grid of the admissible set with a nonhomogeneous distribution of the sampling points (that can be viewed in Fig. 7). For each point $\left(\delta_{\mathcal{M}}, \delta_{\mathcal{K}}\right)$ in the grid, $c_{\mathcal{H}} \mapsto$ $J_{s}\left(\delta_{\mathcal{M}}, \delta_{\mathcal{K}}, c_{\mathcal{H}}\right)$ is maximized yielding the optimal value $c_{\mathcal{H}}^{\star}\left(\delta_{\mathcal{M}}, \delta_{\mathcal{K}}\right)$. Figure 7 displays the graph of function $\left(\delta_{\mathcal{M}}\right.$, $\left.\delta_{\mathcal{K}}\right) \mapsto J_{s}\left(\delta_{\mathcal{M}}, \delta_{\mathcal{K}}, c_{\mathcal{H}}^{\star}\left(\delta_{\mathcal{M}}, \delta_{\mathcal{K}}\right)\right)$ calculated at the sampling points of the grid. It can be seen that there is a quasi-symmetry with respect to $\delta_{\mathcal{M}}=\delta_{\mathcal{K}}$ axis. It can then be deduced the optimal solution, for which $\delta_{\mathcal{M}}^{\text {opt }}=0.13$ and $\delta_{\mathcal{K}}^{\text {opt }}=0.11$

Concerning the calculation of the objective function, for each sampling point of the grid, the value of the objective function $J_{s}$ is estimated using $n_{\text {sim }}$ Monte-Carlo simulations. Figure 8 presents the convergence of the objective function $J_{s}$ as a function of $n_{\text {sim }}$ evaluated at the optimal point $\left(\delta_{\mathcal{M}}^{\mathrm{opt}}, \delta_{\mathcal{K}}^{\mathrm{opt}}, c_{\mathcal{H}}^{\mathrm{opt}}\right)$. It can be seen that, for a reasonable precision of $J_{s}$ (of about 0.01 ), convergence is reached quite fast for $n_{\text {sim }}=40$, which can be considered as a good compromise between the numerical cost and the accuracy (it would make little sense to carry out a very fine statistical estimation if not exploring the parameter space with a sufficiently fine grid - and vice versa).

Concerning the computational cost, for each independent realization of random matrices $[\mathcal{M}]$ and $[\mathcal{K}]$ in Eq. (11), the matrix equation is diagonalized (solving the generalized eigenvalue problem of reduced dimension associated with the conservative dynamical system) for obtaining an efficient resolution over (1) the frequency sampling and (2) the sampling of parameter $c_{\mathcal{H}}$.

Confidence regions of the random FRFs and experimental comparison. The confidence region (corresponding

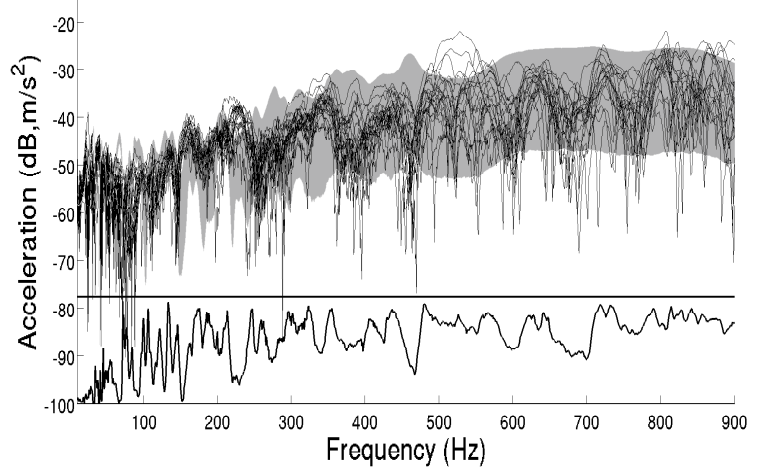

Fig. 9 Observation 1: experimental FRF measurements (black lines), random FRF using the identified C-SROM (gray region), and overlap function OVL (black line underneath)

to a probability level of $95 \%$ ) of each FRF is computed 


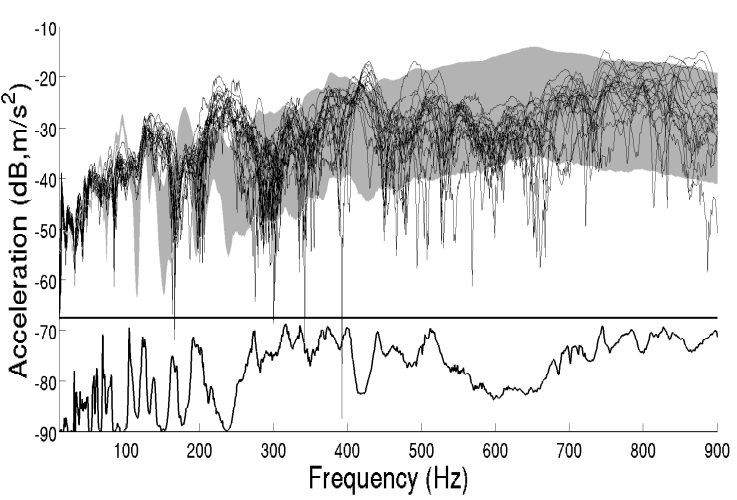

Fig. 10 Observation 2: experimental FRF measurements (black lines), random FRF using the identified C-SROM (gray region), and overlap function OVL (black line underneath)

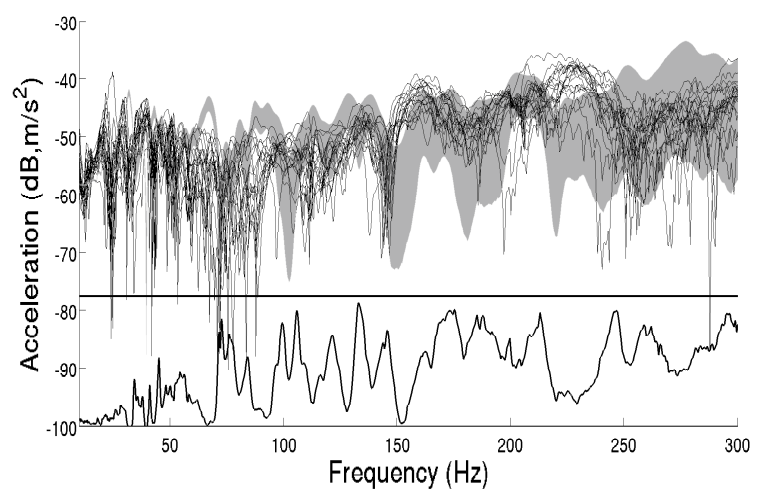

Fig. 11 Observation 1, zoom into band $\mathcal{B}_{\mathcal{L}} \cup \mathcal{B}_{\mathcal{M}}$ : experimental FRF measurements (black lines), random FRF using the identified C-SROM (gray region), and overlap function OVL (black line underneath)

by using the identified C-SROM. A convergence analysis of the confidence region with respect to the number $n_{\text {sim }}$ of realizations in the Monte-Carlo simulation method has been performed. A good convergence of the confidence region is reached for $n_{\text {sim }}=10,000$. The corresponding values of objective functions $J_{s, 1}$ and $J_{s, 2}$ for the identified C-SROM are $J_{s, 1}=0.62$ and $J_{s, 2}=0.56$, which yields $J_{s}=0.59$ (the statistical estimation is done with $\left.n_{\text {sim }}=10,000\right)$. The results for observation 1 and observation 2 are displayed in Figs. 9 and 10. On each figure, it can be seen the confidence region, the 20 experimental measurements, and the OVL function $\omega \mapsto$ $\operatorname{OVL}\left(U_{j}^{c}(\omega), U_{j}^{e}(\omega)\right)$ defined by Eq. (87). This OVL function is plotted between two horizontal lines. The lower horizontal line corresponds to the value 0 and the upper one to the value 1 . In Figs. 9 and 10, it can be seen that the C-SROM does not perfectly represent most of the experimental FRFs in the LF and MF bands. This can be explained by the discrepancies of the C-NROM with respect to the experiments and by the too narrow

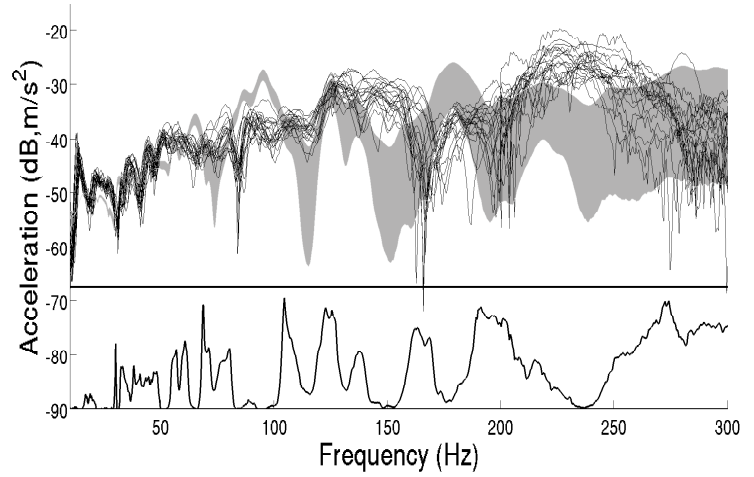

Fig. 12 Observation 2, zoom into band $\mathcal{B}_{\mathcal{L}} \cup \mathcal{B}_{\mathcal{M}}$ : experimental FRF measurements (black lines), random FRF using the identified C-SROM (gray region), and overlap function OVL (black line underneath)

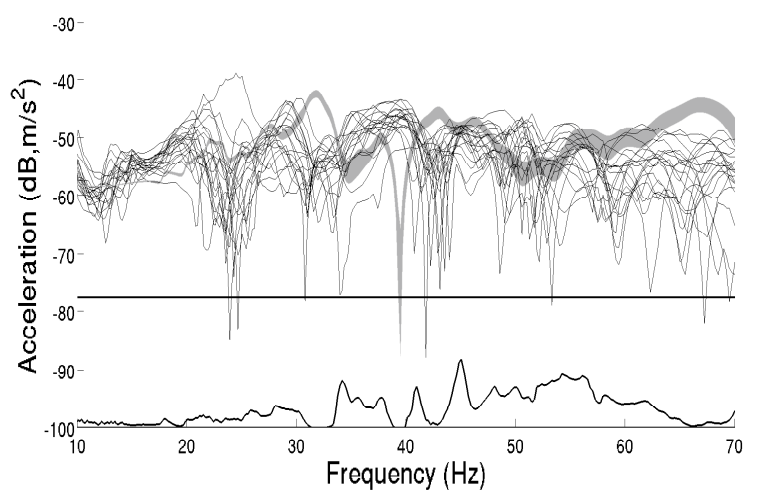

Fig. 13 Observation 1, zoom into band $\mathcal{B}_{\mathcal{L}}$ : experimental FRF measurements (black lines), random FRF using the identified C-SROM (gray region), and overlap function OVL (black line underneath)

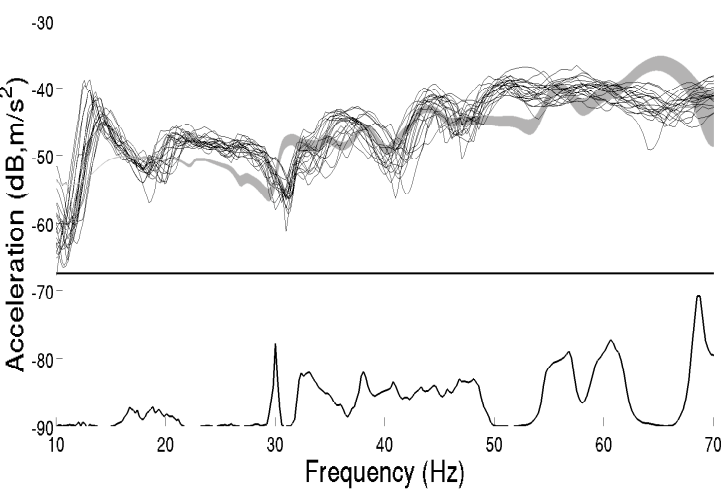

Fig. 14 Observation 2, zoom into band $\mathcal{B}_{\mathcal{L}}$ : experimental FRF measurements (black lines), random FRF using the identified C-SROM (gray region), and overlap function OVL (black line underneath)

confidence regions provided by the $\mathrm{C}-\mathrm{SROM}$ in the LF band (and to a lesser extent in the MF band). This can 
be better seen in Figs. 11 and 12 that present a zoom of Figs. 9 and 10 into band $\mathcal{B}_{\mathcal{L}} \cup \mathcal{B}_{\mathcal{M}}$, and in Figs. 13 and 14 that present a zoom of Figs. 9 and 10 into band $\mathcal{B}_{\mathcal{L}}$. It can also be seen that OVL function confirms the not perfectly correct prediction of the C-SROM in the LF and MF bands. The reason why such a one-level stochastic model of uncertainties is not sufficient for predicting the confidence regions in all the frequency band is that the effects of uncertainties on the FRFs are not homogeneous in the frequency band. It should be noted that, with this one-level stochastic ROM, if one would want to obtain broader confidence regions in the LF band, one could use greater values for the dispersion hyperparameters but in such a case, one would consequently obtain too broad confidence regions in the $\mathrm{HF}$ band. The introduction of a multilevel stochastic ROM allows for improving the prediction as demonstrated in the next section.

\subsection{Multilevel nominal ROM and multilevel stochastic} ROM

In a first step, a deterministic analysis is carried out in order to find suitable filtering parameters, which affect the reduction of the dimension of the model. In fact, this deterministic analysis is not sufficient in itself, as the final objective is the experimental identification of the ML-SROM. Consequently, in a second step, all the filtering parameters defining the multilevel ROB and all the dispersion hyperparameters are simultaneously identified. To this end, first, a temporary choice of filtering parameters defining the ML-NROM is done, based on the deterministic analysis. Then, a sensitivity analysis with respect to the dispersion hyperparameters allows for decreasing the number of dispersion hyperparameters to be identified. Based on this assumption, a $3 D$ coarse grid allows for finding initial values for the dispersion hyperparameters. All the other parameters being fixed, the filtering parameters of the HF band and the dispersion hyperparameter of the HF band are simultaneously identified in a precise way. Finally, defining the values of the other filtering parameters independently of the global optimization problem, the remaining dispersion hyperparameters are identified at a low cost. Using the identified ML-SROM, the confidence regions of the random FRFs are statistically estimated and are then compared to the experimental measurements.

\subsubsection{First step: $M L-N R O M$}

Convergence analysis of $J_{d}$ as a function of dimension $n_{t}$ deduced from a first filtering. For constructing the multilevel ROM, the first step consists in defining the filtering of local displacements, which is devoted to the reduction of the final dimension $n_{t}$ of the proposed ROM. This step corresponds to either Eq. (49) or Eq. (59). It depends on the maximum degree $d_{\mathcal{H}}$ of the polynomials of the reduced kinematics of mass matrix $\left[\mathbb{M}^{r}\right]$, on truncation order $\nu_{\mathcal{H}}$ that is indirectly related to the upper bound of the frequency band (through the value $\sqrt{\sigma_{\nu_{\mathcal{H}}}^{t}} / 2 \pi$ in which $\sigma_{\alpha}^{t}$ is the eigenvalue of rank $\alpha$ in Eq. (54), involved in the mapping $\mathcal{F}_{2}$ in Eq. (59)) and to the cutoff frequency $f_{\mathcal{H}}^{c}$. It should be noted that since by construction one has $\mathcal{S}_{t} \subseteq \mathcal{S}_{c}$, the frequency $f_{n_{t}}^{t}=\sqrt{\lambda_{n_{t}}^{t}} / 2 \pi$ verifies the inequality $f_{n_{t}}^{t} \leq f_{n}$. Therefore, choosing $f_{\mathcal{H}}^{c}=$ $f_{n}=1,000 \mathrm{~Hz}$ would automatically yield $n_{t}=\nu_{\mathcal{H}}$. The value of $f_{\mathcal{H}}^{c}$ is taken as $925 \mathrm{~Hz}$. It should be noted that the scale- $t$ ROM (given by Eqs. (68) and (69)) yields the same response as the ML-NROM (by construction). Exploring the possible values of parameters $d_{\mathcal{H}}$ and $\nu_{\mathcal{H}}$ allows the construction of the ML-NROM to be adjusted. It is recalled that $\lambda_{\nu_{\mathcal{H}}}^{t} \leq \sigma_{\nu_{\mathcal{H}}}^{t}$. For fixed $d_{\mathcal{H}}$, some sampling points are explored in a segment in which parameter $\nu_{\mathcal{H}}$ verifies

$f_{\mathcal{H}}^{c} \leq \sqrt{\sigma_{\nu \mathcal{H}}^{t}} / 2 \pi \leq 10 \times f_{\mathcal{H}}^{c}$.

Figure 15 presents a plot of function $J_{d}$ with respect to parameters $d_{\mathcal{H}}$ and $\nu_{\mathcal{H}}$, with $d_{\mathcal{H}}$ ranging from 2 to 40 and with the corresponding values of $\nu_{\mathcal{H}}$ deduced from Eq. (88). For $\nu_{\mathcal{H}} \geq 4,000$, it can be seen that the value of $J_{d}$ is only subjected to small fluctuations. It should also be noted that, in general, for a fixed $\nu_{\mathcal{H}}$ between 2,500 and 4,000, the value of $J_{d}$ is greater (i.e. is less good) for a greater value of $d_{\mathcal{H}}$. This is due to the fact that for an increasing value of $d_{\mathcal{H}}$, the value of $\nu_{\mathcal{H}}$ has to increase in order that the quantity $\sqrt{\sigma_{\nu \mathcal{H}}^{t}} / 2 \pi$ reach the upper bound of the frequency band. Indeed, a larger maximum degree $d_{\mathcal{H}}$ means an increasing presence of local displacements, which means more basis vectors kept in the construction of the ROM.

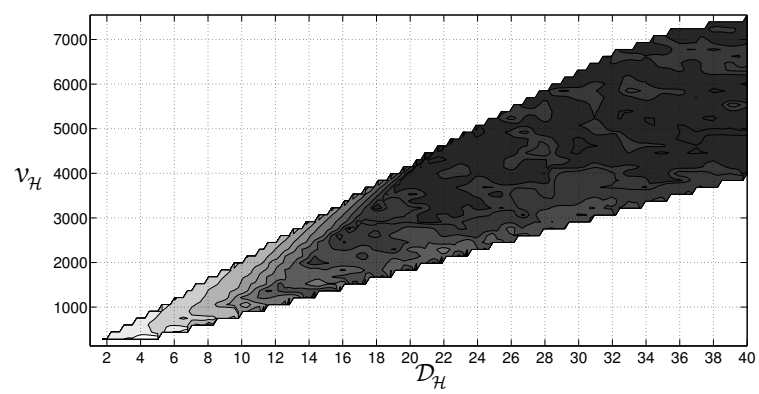

Fig. 15 Plot of function $J_{d}$ with respect to parameters $d_{\mathcal{H}}$ and $\nu_{\mathcal{H}}$ (lighter gray level meaning higher $J_{d}$ )

Figure. 16 shows the convergence of the ML-NROM with respect to its dimension $n_{t}$. For this, the value of $J_{d}$ is plotted as a function of $n_{t}$ for several ROMs (all the sampling points). Likewise, the convergence of the C-NROM is given on the same figure, in order to put into evidence the faster convergence of the ML-NROM towards a value corresponding to a reasonable accuracy, compared to the 


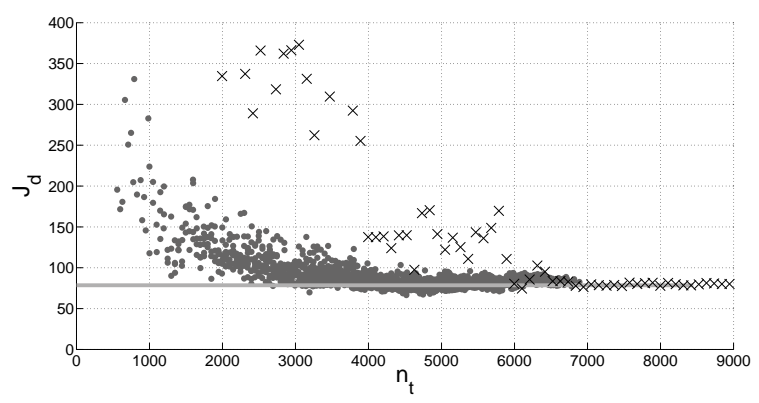

Fig. 16 For the C-NROM, graph of $n \mapsto J_{d}(n)$ (black crosses) and graph of $n \mapsto J_{d}(8,450)$ (horizontal light-gray line). For the ML-NROM, graph of $n_{t} \mapsto J_{d}\left(n_{t}\right)$ (gray dots).

C-NROM. This is explained by the fact that the MLNROM lacks local displacements (and thus has a lower dimension), which are not really important for representing the dynamical responses. For a low maximum degree $d_{\mathcal{H}}$ of the polynomials (and for a low truncation order $\nu_{\mathcal{H}}$ ), the lower dimension $n_{t}$ that is obtained for the $\mathrm{ROM}$ is associated with more important modeling errors. For constructing the ML-NROM, a good compromise between the value of $n_{t}$ and the accuracy associated with the value of $J_{d}$ is sought. In fact, the purpose of the multilevel ROM is to better represent the variabilities of the experimental measurements in the frequency band (i.e. to obtain random FRFs that are able to represent the experimental measurements) and consequently, the identification of parameters $d_{\mathcal{H}}$ and $\nu_{\mathcal{H}}$ depends on the values taken by the dispersion hyperparameters of the MLSROM (coupled problem). The previous exploration of the possible values of parameters $d_{\mathcal{H}}$ and $\nu_{\mathcal{H}}$ is thus not sufficient. Nevertheless, it allows for defining a subregion in which the global identification (taking into account the coupling of the dispersion hyperparameters with the filtering parameters) of the ML-SROM will be restrained. Concerning the computational cost, one sampling point involves solving an eigenvalue problem of dimension $\nu_{\mathcal{H}}$ (associated with Eq. (20) or Eq. (54)). In addition, it implies solving, for each value of the maximum degree $d_{\mathcal{H}}$ of the polynomials, an eigenvalue problem of dimension $n$ (associated with Eq. (30) or Eq. (53)), which can be solved at a lower cost if $d_{\mathcal{H}}$ (and thus column dimension $r_{\mathcal{H}}$ of matrix $\left[N^{t}\right]$ in Eq. (59)) is low, through a SVD (as explained in Remark 2 of Section 3.3). Furthermore, for each value of maximum degree $d_{\mathcal{H}}$ of the polynomials, the matrix $\left[N^{t}\right]$, which is constructed in Eq. (52) within the use of mapping $\mathcal{F}_{2}$ in Eq. (59), can be obtained by extracting the first $r_{\mathcal{H}}$ columns of a matrix $\left[N^{t}\right]$ associated with a reduced kinematics of dimension $r_{\mathcal{H}, \max }$ that satisfies $r_{\mathcal{H}, \max } \geq r_{\mathcal{H}}$ for all considered values of $r_{\mathcal{H}}$. This way, the construction of matrix $\left[B^{\ell}\right]$ and the matrix product with $(m \times n)$ matrix $[\Phi]$ are done once and for all.

Deterministic FRFs and experimental comparisons. Fig-

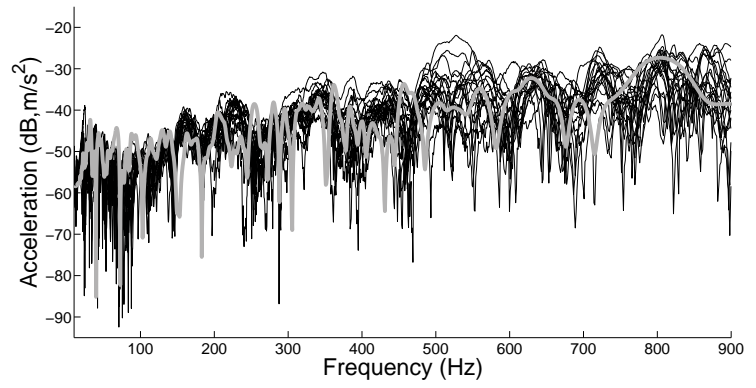

Fig. 17 Observation 1: experimental FRF measurements (black lines), deterministic FRF using the identified MLNROM (gray line)

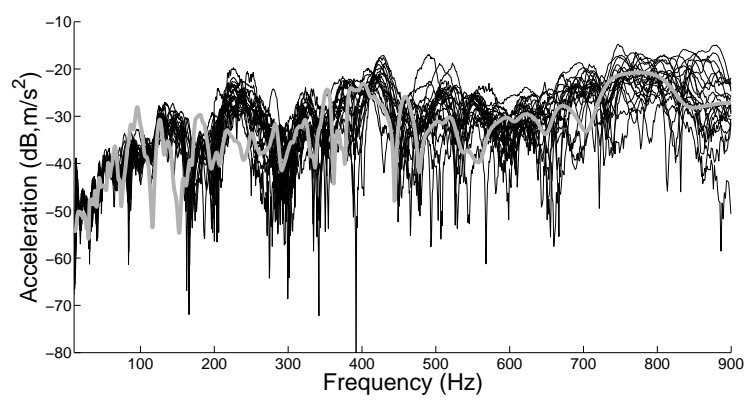

Fig. 18 Observation 2: experimental FRF measurements (black lines), deterministic FRF using the identified MLNROM (gray line)

ures 17 and 18 show the deterministic FRFs obtained using the ML-NROM with $d_{\mathcal{H}}=34$ and $\nu_{\mathcal{H}}=4,250$, which are the estimated optimal values obtained by solving the global stochastic optimization problem presented in the next section. It can be seen that the ML-NROM, with reduced dimension $n_{t}=4,232$, yields a satisfactory prediction with respect to that of the C-NROM.

\subsubsection{Second step: $M L-S R O M$}

Global experimental identification of all the parameters defining the $M L$-SROM. For the inverse identification of the ML-SROM, the global stochastic optimization problem requires identifying the successive input parameters (filtering parameters) of function $\mathcal{F}_{1}$ in Eqs. (48), (49), and (50) used for defining the ML-NROM. Based on the ML-NROM, the ML-SROM is constructed upon dispersion hyperparameters $\delta_{K}^{\mathcal{L}}, \delta_{M}^{\mathcal{L}}, \delta_{K}^{\mathcal{M}}, \delta_{M}^{\mathcal{M}}, \delta_{K}^{\mathcal{H}}$, and $\delta_{M}^{\mathcal{H}}$ that also have to be identified. The random reduced damping matrix $[\mathbf{D}]$ of the ML-SROM is defined in Section 5.1.4 and consequently, this random matrix is not controlled by dispersion hyperparameters $\delta_{D}^{\mathcal{L}}, \delta_{D}^{\mathcal{M}}$, and $\delta_{D}^{\mathcal{H}}$. The cutoff frequencies $f_{\mathcal{L}}^{c}$ and $f_{\mathcal{M}}^{c}$ are chosen as the upper bounds of bands $\mathcal{B}_{\mathcal{L}}$ and $\mathcal{B}_{\mathcal{M}}$, that is to say $f_{\mathcal{L}}^{c}=$ $70 \mathrm{~Hz}$ and $f_{\mathcal{M}}^{c}=300 \mathrm{~Hz}$. It is recalled that $f_{\mathcal{H}}^{c}=925 \mathrm{~Hz}$. To sum up, in addition to $c_{\mathcal{H}}$ and to the 6 dispersion hyperparameters, the ML-SROM is defined by the 6 fil- 
tering parameters, $\left(d_{\mathcal{H}}, \nu_{\mathcal{H}}\right),\left(d_{\mathcal{M}}, \nu_{\mathcal{M}}\right)$, and $\left(d_{\mathcal{L}}, \nu_{\mathcal{L}}\right)$.

Construction of a first version of the ML-NROM. First, from the numerical exploration of parameters $\left(d_{\mathcal{H}}, \nu_{\mathcal{H}}\right)$ in a deterministic framework as described in latter section (see also Fig. 15), a first version of the scale- $t$ ROM, given by $d_{\mathcal{H}}=20$ and $\nu_{\mathcal{H}}=3,900$, is chosen. Then, successive numerical explorations of the parameters $\left(d_{\mathcal{M}}, \nu_{\mathcal{M}}\right)$ and $\left(d_{\mathcal{L}}, \nu_{\mathcal{L}}\right)$ are carried out in a similar manner by using the scale- $\mathcal{L} \mathcal{M}$ and scale- $\mathcal{L}$ ROMs (these ROMs were introduced in Section 4.2.3). These explorations yield $\left(d_{\mathcal{M}}=12, \nu_{\mathcal{M}}=800\right)$ and $\left(d_{\mathcal{L}}=6, \nu_{\mathcal{L}}=275\right)$. It should be noted that the coupling with the dispersion hyperparameters to be identified is, at this step, not taken into account.

Sensitivity analysis of the $M L-S R O M$ with respect to the dispersion hyperparameters. A sensitivity analysis of the ML-SROM associated with latter definition of the MLNROM is carried out. It shows that for a given scale $\mathcal{S}$ equal to $\mathcal{L}, \mathcal{M}$ or $\mathcal{H}$, the influence of parameters $\delta_{M}^{\mathcal{S}}$ and $\delta_{K}^{\mathcal{S}}$ (associated with the statistical dispersion of reduced mass and stiffness matrices) is similar. Therefore, only 3 dispersion hyperparameters $\delta^{\mathcal{L}}, \delta^{\mathcal{M}}$, and $\delta^{\mathcal{H}}$ have to be identified: $\delta^{\mathcal{L}}=\delta_{K}^{\mathcal{L}}=\delta_{M}^{\mathcal{L}}, \delta^{\mathcal{M}}=\delta_{K}^{\mathcal{M}}=\delta_{M}^{\mathcal{M}}$, and $\delta^{\mathcal{H}}=\delta_{K}^{\mathcal{H}}=\delta_{M}^{\mathcal{H}}$.

First identification of the hyperparameters of the $M L$ SROM using a coarse $3 D$ grid. A first identification is carried out using a coarse $\delta^{\mathcal{L}} \times \delta^{\mathcal{M}} \times \delta^{\mathcal{H}}$ grid, for which the boundaries are deduced from the previous sensitivity analysis. The $3 D$ grid is constituted of 540 sampling points, defined by the cartesian product of the following sets $\delta^{\mathcal{L}} \times \delta^{\mathcal{M}} \times \delta^{\mathcal{H}}$ :

$$
\begin{aligned}
& \boldsymbol{\delta}^{\mathcal{L}}=(0.15,0.20,0.25,0.30,0.35,0.40,0.45,0.50, \ldots \\
& 0.55,0.60) \\
& \boldsymbol{\delta}^{\mathcal{M}}=(0.10,0.15,0.20,0.25,0.30,0.35,0.40, \ldots \\
& 0.45,0.50) \\
& \quad \boldsymbol{\delta}^{\mathcal{H}}=(0.05,0.07,0.09,0.11,0.13,0.15) .
\end{aligned}
$$

As for the C-SROM, only $n_{\text {sim }}=40$ Monte-Carlo simulations are used for estimating the objective function $J_{s}$ associated with each sampling point. The optimal point found is $\delta^{\mathcal{L}}=0.25, \delta^{\mathcal{M}}=0.25, \delta^{\mathcal{H}}=0.11$, which is sufficiently far from the grid boundary. Concerning the computational cost, similarly to the C-SROM, for each independent realization of random matrices $[\mathbf{M}]$ and $[\mathbf{K}]$ in Eq. (82), the matrix equation is diagonalized, by solving an eigenvalue problem of dimension $n_{t}$. In addition, damping parameter $c_{\mathcal{H}}$ is identified for each sampling point. It should be noted that, compared to dimension $n=8,450$ of the classical ROM, the final dimension $n_{t}=4,232$ of the identified multilevel ROM allows for obtaining a nonnegligible gain of about a factor of ten (the complexity of a full eigenvalue problem being ap- proximatively cubic).

Precise and simultaneous identification of the filtering parameters of the $H F$ band and of the dispersion hyperparameter of the $H F$ band. We are now interested in adjusting the values of parameters $\left(d_{\mathcal{H}}, \nu_{\mathcal{H}}\right)$, responsible for the dimension $n_{t}$ of the ML-NROM. Supposing these filtering parameters are sufficiently big, the choice of their values does not influence the random FRFs in the LF and MF bands (which have already converged with respect to them). The influence of parameter $\delta^{\mathcal{M}}$ (and especially of parameter $\delta^{\mathcal{L}}$ ) is not preponderant for the random response in the $\mathrm{HF}$ band. Consequently, fixing the values of $\delta^{\mathcal{L}}$ and $\delta^{\mathcal{M}}$ that have been identified in the coarse $3 D$ grid, the ML-SROM is identified with respect to filtering parameters $\left(d_{\mathcal{H}}, \nu_{\mathcal{H}}\right)$ and to dispersion hyperparameter $\delta^{\mathcal{H}}$, simultaneously. It should be noted that the identification does not consist, at this step, in picking the parameters that maximize the objective function $J_{s}$. Rather, it consists in finding a set of parameters for which $J_{s}$ is close to its maximum but under the constraint of a reasonably small dimension $n_{t}$ for the ROM. Anyway, it should be recalled that $J_{s}$ is only estimated using $n_{\text {sim }}=40$ realizations. After this step, the parameters that are chosen are $d_{\mathcal{H}}=34, \nu_{\mathcal{H}}=4,250$, and $\delta^{\mathcal{H}}=0.078$.

Final stage for the global identification of the ML-SROM. Parameters $\left(d_{\mathcal{L}}, \nu_{\mathcal{L}}\right),\left(d_{\mathcal{M}}, \nu_{\mathcal{M}}\right), \delta^{\mathcal{L}}$, and $\delta^{\mathcal{M}}$ remain to be identified. In order to avoid a 6-dimensional costly optimization problem, the 4 filtering parameters are left unchanged. It should be noted that these parameters control the overlap of subspaces $\mathcal{S}_{\mathcal{L}}, \mathcal{S}_{\mathcal{M}}$, and $\mathcal{S}_{\mathcal{H}}$ of the multilevel ROM, associated with the LF-, MF-, and HF-type displacements. Also, if parameters $\left(d_{\mathcal{L}}, \nu_{\mathcal{L}}\right)$ and $\left(d_{\mathcal{M}}, \nu_{\mathcal{M}}\right)$ tended towards infinity, then there would be no overlap of subspaces $\mathcal{S}_{\mathcal{L}}, \mathcal{S}_{\mathcal{M}}$, and $\mathcal{S}_{\mathcal{H}}$. Qualitatively, subspace $\mathcal{S}_{\mathcal{L}}$ is supposed to be composed of long-wavelength global displacements, without numerous local displacements. Therefore, based on these physical considerations, it is more suitable to force the values of filtering parameters $\left(d_{\mathcal{L}}, \nu_{\mathcal{L}}\right)$, and $\left(d_{\mathcal{M}}, \nu_{\mathcal{M}}\right)$ outside the global stochastic identification problem of the ML-SROM. Then, parameters $\delta^{\mathcal{L}}$ and $\delta^{\mathcal{M}}$ of the ML-SROM are identified by estimating objective function $J_{s}$ at the sampling points of a given $2 D$ grid, the other parameters being fixed. After this final stage, the identified values are $\delta^{\mathcal{L}}=$ 0.4 and $\delta^{\mathcal{M}}=0.22$. For the identified ML-SROM, the values of objective functions $J_{s, 1}$ and $J_{s, 2}$ are $J_{s, 1}=0.65$ and $J_{s, 2}=0.64$, hence $J_{s}=0.65$. Such a value corresponds to a nonnegligible improvement with respect to the C-SROM.

Confidence regions of the random FRFs and experimental comparisons. The random FRFs associated with the identified ML-SROM are plotted in Figs. 19 and 20. It can be seen that, despite the discrepancies of the ML- 


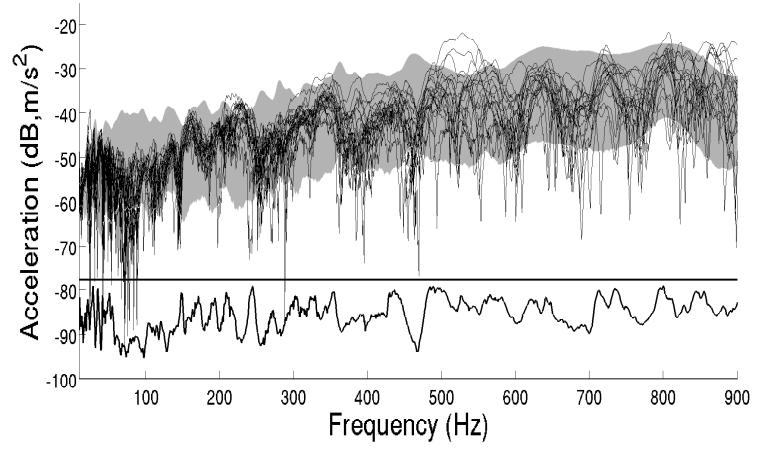

Fig. 19 Observation 1: experimental FRF measurements (black lines), random FRF using the identified ML-SROM (gray region), and OVL function (black line underneath)

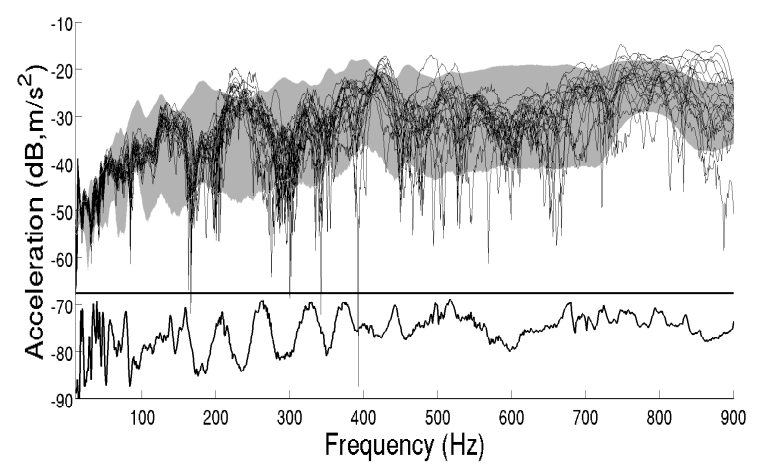

Fig. 20 Observation 2: experimental FRF measurements (black lines), random FRF using the identified ML-SROM (gray region), and OVL function (black line underneath)

NROM (which are similar to those of the C-NROM), the ML-SROM is able to represent most of the experimental FRFs (unlike the C-SROM).

This improvement is due to the increased flexibility of the ML-SROM with respect to the C-SROM, particularly concerning the capability to adapt the level of uncertainties to each frequency band. It should be noted that, in general, on one hand the variability of the real system is low in the LF band and that, on the other hand, the robustness of the computational models is better in this band. In the present case, in the LF and MF bands, the modeling errors are more important than the level of variabilities of the real system, hence the large confidence intervals provided by the ML-SROM in these bands. Figures 21 and 22 display a zoom of Figs. 19 and 20 into band $\mathcal{B}_{\mathcal{L}} \cup \mathcal{B}_{\mathcal{M}}$ and, Figs. 23 and 24 display a zoom of Figs. 19 and 20 into band $\mathcal{B}_{\mathcal{L}}$. It can be seen that the OVL function confirms the improved prediction of the ML-SROM in the LF and MF bands.

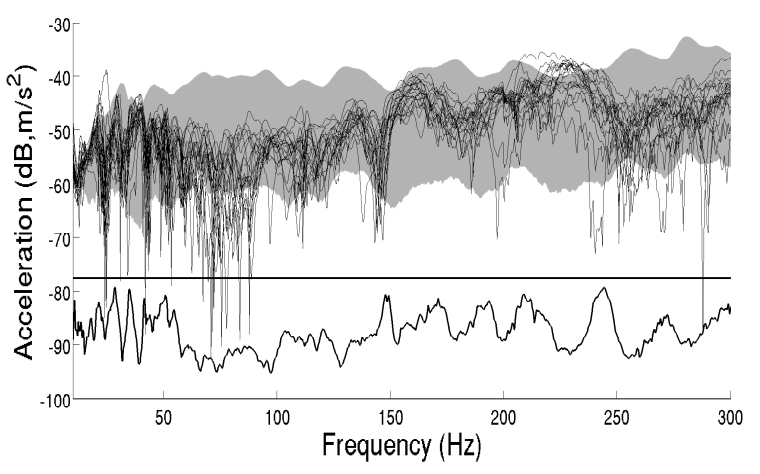

Fig. 21 Observation 1, zoom into band $\mathcal{B}_{\mathcal{L}} \cup \mathcal{B}_{\mathcal{M}}$ : experimental FRF measurements (black lines), random FRF using the identified ML-SROM (gray region), and OVL function (black line underneath)

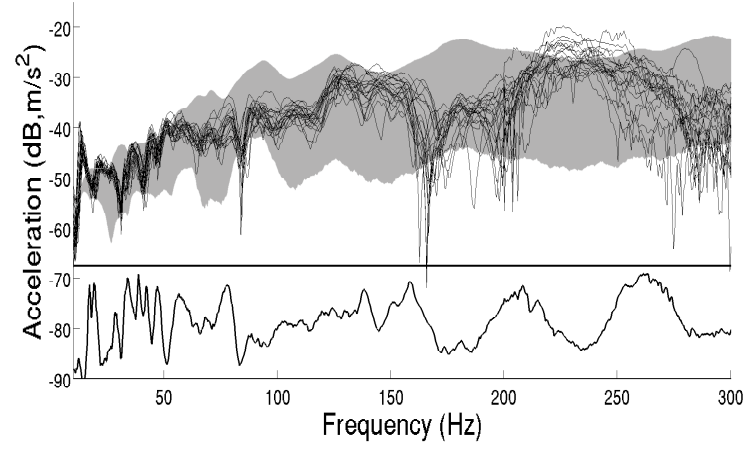

Fig. 22 Observation 2, zoom into band $\mathcal{B}_{\mathcal{L}} \cup \mathcal{B}_{\mathcal{M}}$ : experimental FRF measurements (black lines), random FRF using the identified ML-SROM (gray region), and OVL function (black line underneath)

\subsection{Sensitivity analysis}

\subsubsection{Proposed $M L-S R O M$}

A sensitivity analysis of the ML-SROM with respect to the dispersion hyperparameters is presented. The objective of such a sensitivity analysis is to quantify the role played by each dispersion hyperparameter that is related to a given type of displacements. Using the identified parameters of the ML-SROM and successively setting to zero 2 dispersion hyperparameters out of the 3 hyperparameters $\delta^{\mathcal{L}}, \delta^{\mathcal{M}}, \delta^{\mathcal{H}}$ allow for quantifying the individual contribution of each scale $\mathcal{L}, \mathcal{M}, \mathcal{H}$ of displacements in the random responses. The random responses are obtained by using the identified ML-SROM and by setting, successively, $\delta^{\mathcal{M}}=\delta^{\mathcal{H}}=0$ (see Fig. 25 for observation 1 and Fig. 28 for observation 2), $\delta^{\mathcal{L}}=\delta^{\mathcal{H}}=0$ (see Fig. 26 for observation 1 and Fig. 29 for observation 2), and $\delta^{\mathcal{L}}=\delta^{\mathcal{M}}=0$ (see Fig. 27 for observation 1 and Fig. 30 for observation 2 ). In each figure, the vertical lines indi- 


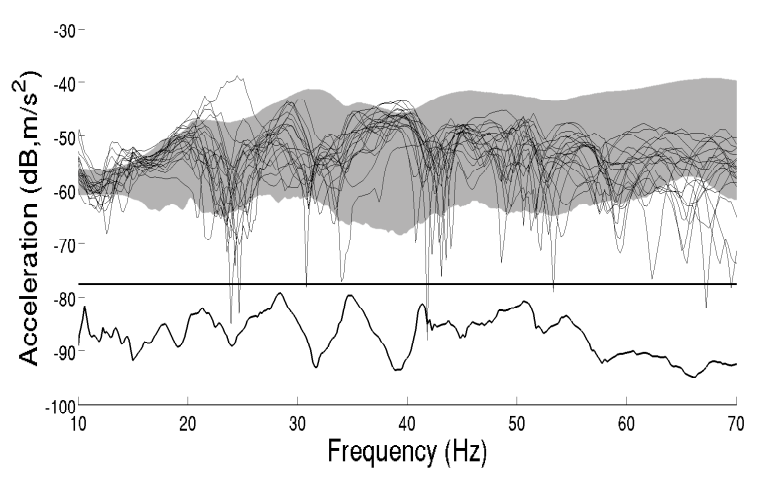

Fig. 23 Observation 1, zoom into band $\mathcal{B}_{\mathcal{L}}$ : experimental FRF measurements (black lines), random FRF using the identified ML-SROM (gray region), and OVL function (black line underneath)

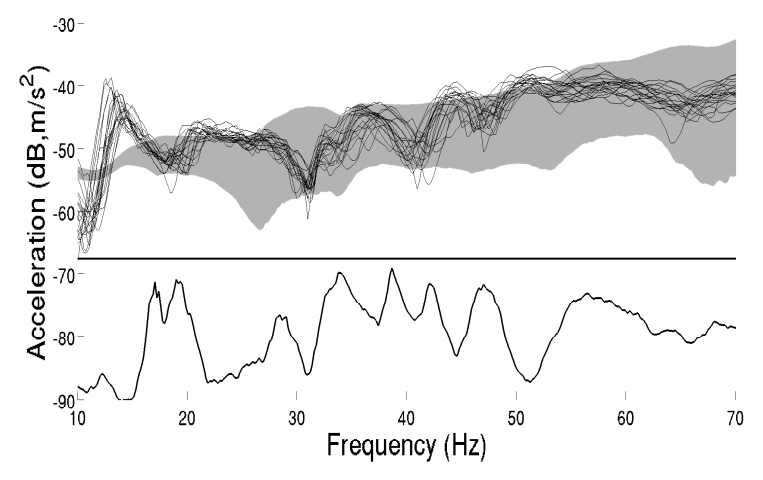

Fig. 24 Observation 2, zoom into band $\mathcal{B}_{\mathcal{L}}$ : experimental FRF measurements (black lines), random FRF using the identified ML-SROM (gray region), and OVL function (black line underneath)

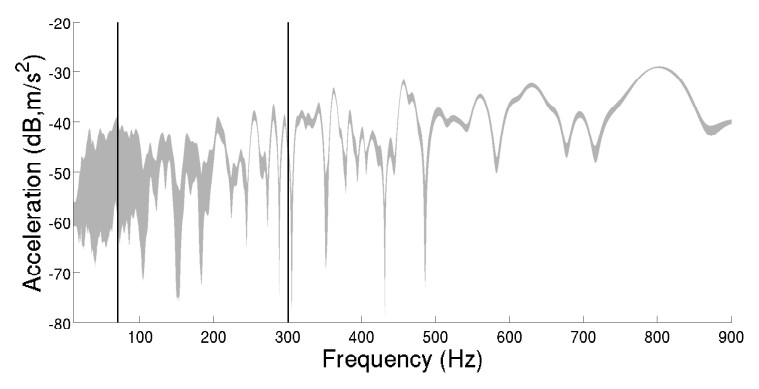

Fig. 25 Observation 1: random FRF using the identified ML-SROM but for which $\delta^{\mathcal{M}}=\delta^{\mathcal{H}}=0$ is imposed.

cate the boundaries between the LF, MF, and HF bands. Figures 27 and 30 show, for instance, that adding uncertainties to the HF-type displacements yields the presence of uncertainties in the LF and MF bands. It is explained by the fact that, since $d_{\mathcal{H}}$ is greater than $d_{\mathcal{M}}$, some HF-

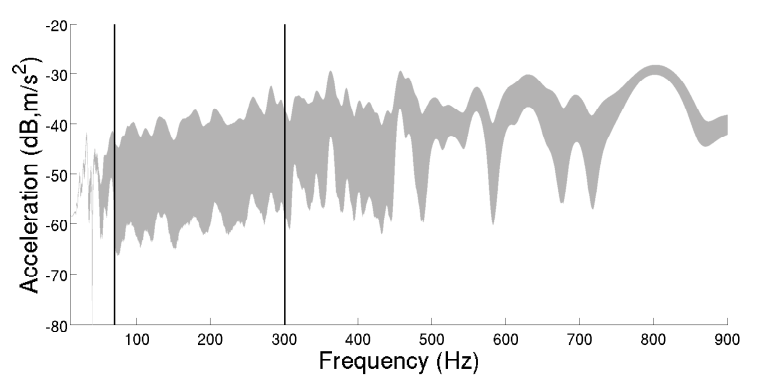

Fig. 26 Observation 1: random FRF using the identified ML-SROM but for which $\delta^{\mathcal{L}}=\delta^{\mathcal{H}}=0$ is imposed.

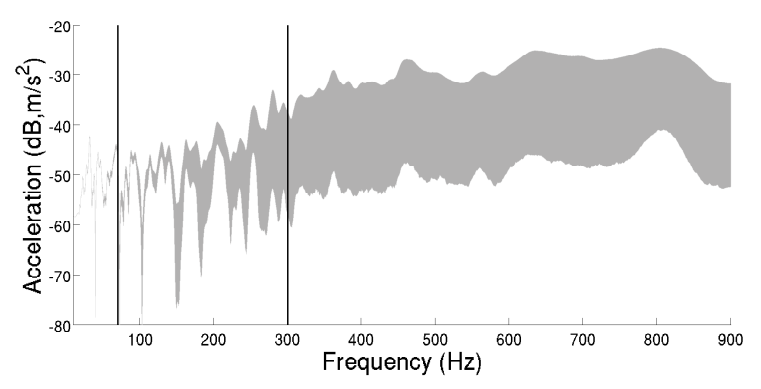

Fig. 27 Observation 1: random FRF using the identified ML-SROM but for which $\delta^{\mathcal{L}}=\delta^{\mathcal{M}}=0$ is imposed.

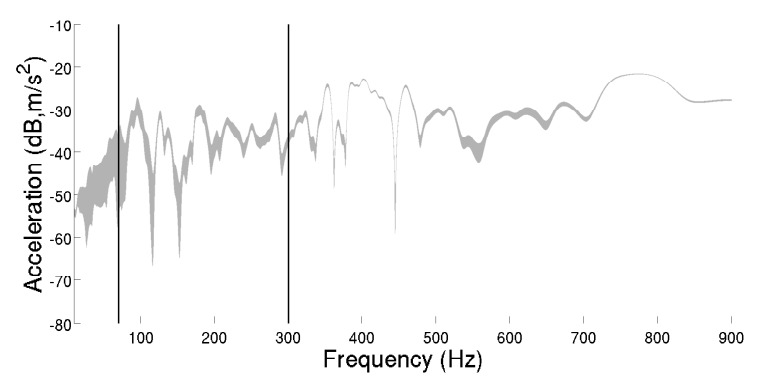

Fig. 28 Observation 2: random FRF using the identified ML-SROM but for which $\delta^{\mathcal{M}}=\delta^{\mathcal{H}}=0$ is imposed.

type displacements are likely to be present in the LF and MF bands.

However, despite the absence of MF-type displacements in the HF band, Figs. 26 and 29 show, for instance, that adding uncertainties to the MF-type displacements yields the presence of uncertainties in the HF band. This is due to the fact that the LF-, MF-, and HF-type displacements are not orthogonal with respect to the stiffness matrix.

\subsubsection{Naive $M L-S R O M$}

A sensitivity analysis is carried out by using a naive ML-SROM that is defined as follows. The vector basis associated with subspace $\mathcal{S}_{\mathcal{L}}$ is given by the 149 elastic 


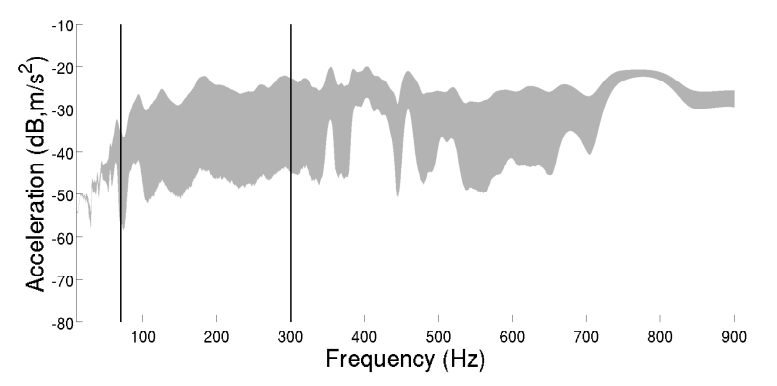

Fig. 29 Observation 2: random FRF using the identified ML-SROM but for which $\delta^{\mathcal{L}}=\delta^{\mathcal{H}}=0$ is imposed.

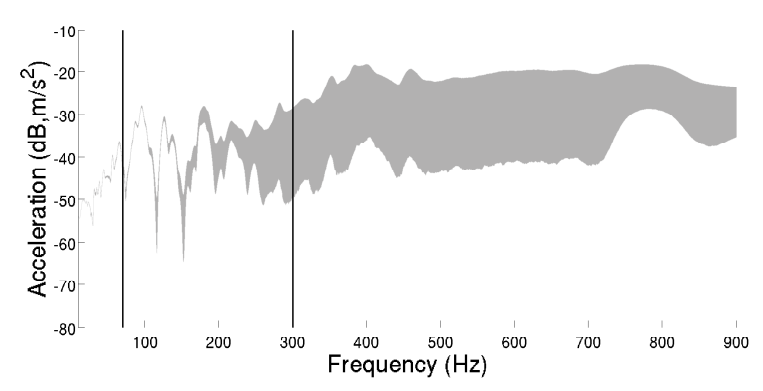

Fig. 30 Observation 2: random FRF using the identified ML-SROM but for which $\delta^{\mathcal{L}}=\delta^{\mathcal{M}}=0$ is imposed.

modes that belong to frequency band $\mathcal{B}_{\mathcal{L}}$, the vector basis associated with subspace $\mathcal{S}_{\mathcal{M}}$ is given by the 1,202 elastic modes that belong to frequency band $\mathcal{B}_{\mathcal{M}}$, and the vector basis associated with subspace $\mathcal{S}_{\mathcal{H}}$ is given by the next 7,099 elastic modes. Note that this ML-SROM corresponds to choosing filtering parameters $\left(d_{\mathcal{L}}, \nu_{\mathcal{L}}\right)$, $\left(d_{\mathcal{M}}, \nu_{\mathcal{M}}\right)$, and $\left(d_{\mathcal{H}}, \nu_{\mathcal{H}}\right)$ as going to infinity (no filtering). The sensitivity analysis of this stochastic ROM is carried out using the same set of combinations of the dispersion hyperparameters as in the previous section. Figures 31, 32, and 33 depict the random FRFs obtained for observation 1 while Figs. 34, 35, and 36 depict the random FRFs obtained for observation 2 . It can be seen that, for this naive ML-SROM, the introduction of uncertainties for one given vector basis induces the presence of uncertainties for the corresponding frequency band, while practically none elsewhere. This stochastic ROM is thus not well adapted for modeling uncertainties of a complex dynamical system for which the LF, MF, and $\mathrm{HF}$ vibration regimes overlap.

\subsection{Remark concerning the use of the proposed} multilevel ROM

The filtering parameters allow for separating the different regimes of vibration (LF, MF, and HF), which can exist in the frequency band of analysis. These regimes can directly be viewed by using the deterministic com-

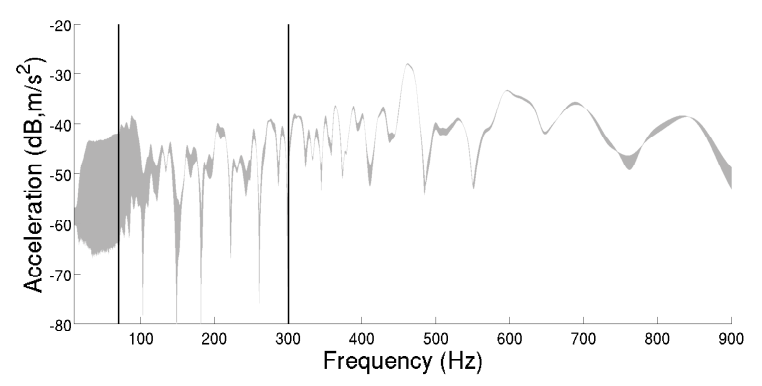

Fig. 31 Observation 1: random FRF using the naive MLSROM for which $\delta^{\mathcal{M}}=\delta^{\mathcal{H}}=0$ is imposed.

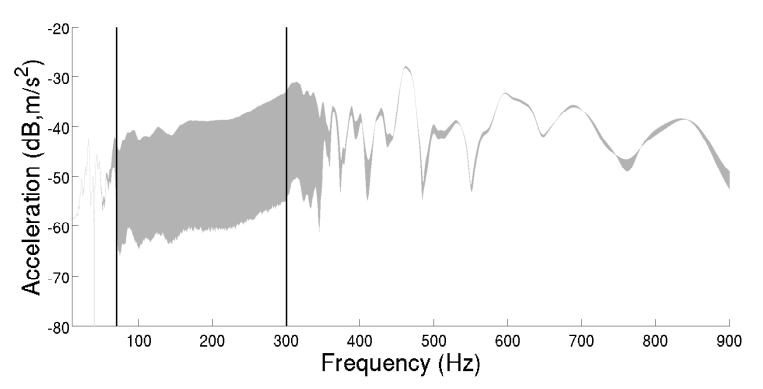

Fig. 32 Observation 1: random FRF using the naive MLSROM for which $\delta^{\mathcal{L}}=\delta^{\mathcal{H}}=0$ is imposed.

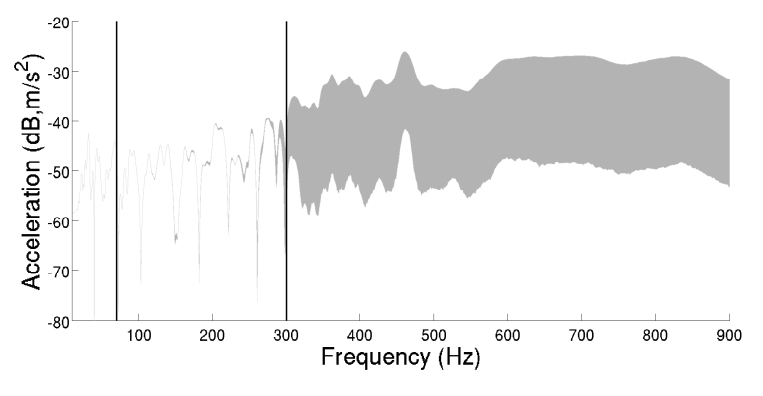

Fig. 33 Observation 1: random FRF using the naive MLSROM for which $\delta^{\mathcal{L}}=\delta^{\mathcal{M}}=0$ is imposed.

putational model for which the classical ROM is constructed as the first step of the multilevel ROM and consequently, allows for analyzing the dynamical behaviors. The filtering parameters are strongly connected to all the geometrical and mechanical properties, but do not induce some problems with respect to the prediction objectives, taking into account the proposed methodology that allows for fixing the values of the filtering parameters without using experiments. The hyperparameters that are introduced in the probabilistic models of uncertainties can either be used for performing a sensitivity analysis with respect to the level of uncertainties if experiments are not available or be identified if experiments are available. In this last case, for a class of dynamical systems (such as a class of popular automobiles corre- 


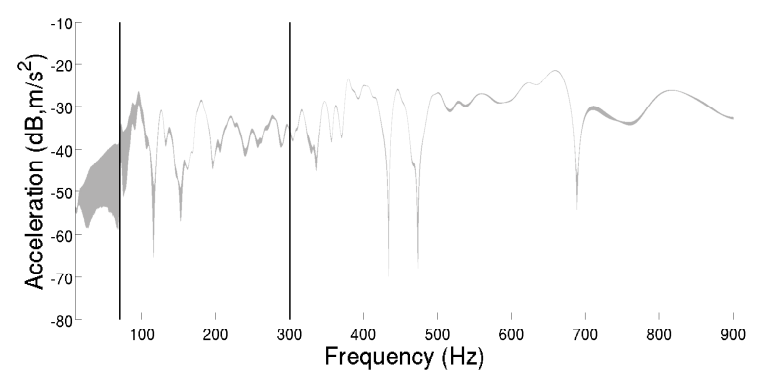

Fig. 34 Observation 2: random FRF using the naive MLSROM for which $\delta^{\mathcal{M}}=\delta^{\mathcal{H}}=0$ is imposed.

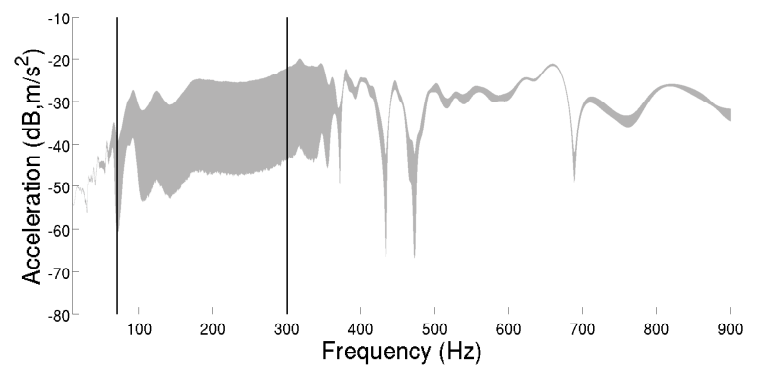

Fig. 35 Observation 2: random FRF using the naive MLSROM for which $\delta^{\mathcal{L}}=\delta^{\mathcal{H}}=0$ is imposed.

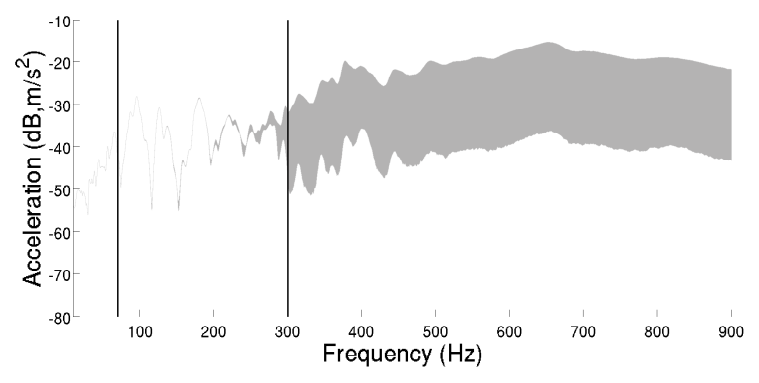

Fig. 36 Observation 2: random FRF using the naive MLSROM for which $\delta^{\mathcal{L}}=\delta^{\mathcal{M}}=0$ is imposed.

sponding to a given level of technologies), the identified hyperparameters can generally be reused (without additional identifications) for similar dynamical systems.

Concerning the numerical cost of the proposed multilevel ROM, for a big computational structural dynamics model (for instance with 10 million of DOFs), which is analyzed over a very broad frequency band (LF, MF, and HF bands), the stochastic analysis (including the identification procedure) requires the introduction of a ROM in order the calculations to be tractable, in particular if such a model is used for robust design. For obtaining the C-SROM, one needs to solve Eq. (2) for the first eigenvalues/eigenvectors (offline stage), which can be costly (large-scale generalized eigenvalue problem).
When the Monte-Carlo method is used a a stochastic solver (that is the case in the paper), each realization of the C-SROM implies solving a full eigenvalue problem of dimension 8,450. For obtaining the ML-SROM, one reuses the eigenvalues/eigenvectors calculated for the C-SROM. All the following calculations devoted to the construction of the multilevel ROM (offline stage) have a negligible cost in comparison to the large-scale generalized eigenvalue problem of Eq. (2). Then, each realization of the ML-SROM implies solving a full eigenvalue problem of dimension 4,232. Due to the roughly cubic complexity of a full eigenvalue problem, the ML-SROM allows for obtaining a speed factor of about 10 compared to the C-SROM. Nevertheless, the ML-SROM requires more parameters to be identified. Still, for this case, the identification of the ML-SROM remains cheaper.

\section{Conclusions}

A general method has been presented for the construction of a multilevel stochastic ROM devoted to the robust dynamical analysis of complex systems over a broad frequency band. The complex systems are constituted of several structural scales. In the low-frequency range, these scales induce the presence of numerous short-wavelength local displacements that are intertwined with the usual long-wavelength global displacements. The proposed multilevel ROM is based on the construction of three orthogonal ROBs whose displacements are either LF-type, either MF-type, or HF-type displacements, and which are associated with the overlap of the LF, the MF, and the HF vibration regimes. The construction of these ROBs relies on a filtering strategy that is based on the introduction of global shape functions for the kinetic energy. It is admitted that the local displacements are more sensitive to uncertainties than the global displacements. Combining the use of the nonparametric probabilistic approach of uncertainties with the constructed multilevel ROB allows for constructing a multilevel stochastic ROM, for which each type of displacements can be assigned to a specific level of uncertainties. As an application, the multilevel stochastic ROM of an automobile is constructed and is identified by using experimental measurements. Such an approach allows for obtaining a smaller dimension for the stochastic ROM as well as an improved prediction, with respect to a classical stochastic ROM.

Acknowledgements This research was supported by Agence Nationale de la Recherche, Contract HiMoDe, ANR-2011BLAN-00378. The authors thank PSA Peugeot Citroën for providing the experimental measurements and the computational model. 


\section{References}

1. Ezvan O, Batou A, Soize C (2015) Multilevel reduced-order computational model in structural dynamics for the low- and mediumfrequency ranges. Comput Struct 160:111-125. doi:http://dx.doi.org/10.1016/j.compstruc.2015.08.007

2. Ezvan O (2016) Multilevel model reduction for uncertainty quantification in computational structural dynamics. Doctoral Thesis, Université Paris-Est, Paris, September 23, 2016.

3. Bathe KJ (1996) Finite element procedures. Prentice Hall, Upper Saddle River, New Jersey.

4. Hughes TJR (2000) The Finite Element Method: Linear Static and Dynamic Finite Element Analysis. Dover Publications, New York.

5. Zienkiewicz OC, Taylor RL (2000) The Finite Element Method, 5th ed. Butterworth-Heinemann, Oxford.

6. Soize C (1982) Medium frequency linear vibrations of anisotropic elastic structures. La Recherche Aérospatiale (English edition) 5:65-87.

7. Bathe KJ, Wilson EL (1976) Numerical methods in the finite element method. Englewood Cliffs, New Jersey: Prentice-Hall.

8. Meirovitch L (1990) Dynamics and Control of Structures. Wiley, New York.

9. Argyris J, Mlejnek HP (1991) Dynamics of Structures. North-Holland, Amsterdam.

10. Geradin M, Rixen D (1997) Mechanical Vibrations, Second edition: Theory and Applications to Structural Dynamics. Wiley, Chichester.

11. Ohayon R, Soize C (1998) Structural acoustics and vibration. Academic Press, San Diego.

12. Craig RR, Kurdila AJ (2006) Fundamentals of Structural Dynamics. Wiley, 2nd Edition, John Wiley and Sons, Hoboken.

13. Bathe KJ (2013) The subspace iteration method - revisited. Comp Struct 126:177-183. doi:http://dx.doi.org/10.1016/j.compstruc.2012.06.002

14. Casciati S, Faravelli L (2014) Quantity vs. quality in the model order reduction (MOR) of a linear dynamical system. Smart Struct Syste 13(1):99-109. doi:http://dx.doi.org/10.12989/sss.2014.13.1.099

15. Lyon RH, DeJong RG (1995) Theory and Application of Statistical Energy Analysis. Butterworths-Heimann, Boston.

16. Langley RS, Bremner P (1999) A hybrid method for the vibration analysis of complex structuralacoustic systems. J Acoust Soc Am 105(3):1657-1671. doi:http://dx.doi.org/10.1121/1.426705

17. LeBot A (2002) Energy transfer for high frequencies in built-up structures. J Sound Vib 250(2):247-275. doi:http://dx.doi.org/10.1006/jsvi.2001.3933

18. Maxit L, Guyader JL (2003) Extension of SEA model to subsystems with non-uniform modal energy distribution. J Sound Vib 265 (2):337-358. doi:http://dx.doi.org/10.1016/S0022-460X(02)01459-1

19. Langley RS, V. Cotoni V (2004) Response variance prediction in the statistical energy analysis of built up systems. J Acoust Soc Am 115(2):706-718. doi:http://dx.doi.org/10.1121/1.1642621

20. Langley RS (2007) On the diffuse field reciprocity relationship and vibrational energy variance in a random subsystem at high frequencies. J Acoust Soc Am 121(2): 913-921. doi:http://dx.doi.org/10.1121/1.2409484

21. Cotoni V, Langley R, Shorter P (2008) A statistical energy analysis subsystem formulation using finite element and periodic structure theory. J Sound Vib 318(4-5):1077-1108. doi:http://dx.doi.org/10.1016/j.jsv.2008.04.058
22. Ragnarsson P, Pluymers B, Donders S, W. Desmet W (2010) Subcomponent modelling of input parameters for statistical energy analysis by using a wavebased boundary condition. J Sound Vib 329(1):96-108. doi:http://dx.doi.org/10.1016/j.jsv.2009.08.033

23. Morand HJP (1992) A modal hybridization method for vibroacoustic studies at medium frequencies. J Acoust Soc Am 92(4):2365-2366. doi:http://dx.doi.org/10.1121/1.404855

24. Ladevèze $\mathrm{P}$ (1996) A new computational approach for structure vibrations in the medium frequency range. CR Acad Sci II B-Mec 322(12):849-856.

25. Soize C (1998) Reduced models in the medium frequency range for general dissipative structuraldynamics systems. Eur J Mech A-Solid 17(4):657-685. doi:http://dx.doi.org/10.1016/S0997-7538(99)80027-8

26. Ladevèze $\mathrm{P}$, Arnaud L, Rouch $\mathrm{P}$, Blanzé $\mathrm{C}$ (2001) The variational theory of complex rays for the calculation of medium-frequency vibrations. Eng Computation 18(1-2):193-214. doi:http://dx.doi.org/10.1108/02644400110365879

27. Farhat C, Harari I, Hetmaniuk U (2003) A discontinuous Galerkin method with Lagrange multipliers for the solution of Helmholtz problems in the mid-frequency regime. Comput Method Appl M 192(11-12):1389-1419. doi:http://dx.doi.org/10.1016/S0045-7825(02)00646-1

28. De Bel E, Villon P, Bouillard Ph (2005) Forced vibrations in the medium frequency range solved by a partition of unity method with local information. Int J Numer Meth Eng 62(9):1105-1126. doi:http://dx.doi.org/10.1002/nme.1202

29. Ladevèze $\mathrm{P}$, Chevreuil M (2005) A new computational method for transient dynamics including the low- and the medium-frequency ranges. Int J Numer Meth Eng 64(4):503-527. doi:http://dx.doi.org/10.1002/nme.1379

30. Shorter PJ, Langley RS (2005) Vibro-acoustic analysis of complex systems. J Sound Vib 288(3):669-699. doi:10.1016/j.jsv.2005.07.010

31. Zhang L, Tezaur R, Farhat C (2006) The discontinuous enrichment method for elastic wave propagation in the medium-frequency regime. Int J Numer Meth Eng 66(13):2086-2114. doi:http://dx.doi.org/10.1002/nme.1619

32. Ji L, Mace BR, Pinnington RJ (2006) A modebased approach for the mid-frequency vibration analysis of coupled long- and short-wavelength structures. J Sound Vib 289(1-2):148-170. doi:http://dx.doi.org/10.1016/j.jsv.2005.02.003

33. Soize C (1993) A model and numerical method in the medium frequency range for vibroacoustic predictions using the theory of structural fuzzy. J Acoust Soc Am 94:849-865. doi:http://dx.doi.org/10.1121/1.408186

34. Sarkar A, Ghanem R (2002) Mid-frequency structural dynamics with parameter uncertainty. Comput Method Appl M 191(47-48):5499-5513. doi:http://dx.doi.org/10.1016/S0045-7825(02)00465-6

35. Gagliardini L, Houillon L, Borello G, Petrinelli L (2003) Virtual SEA: Mid-frequency structure-borne noise modeling based on finite element analysis. In: Noise \& Vibration Conference, Traverse City, MI, USA, May 5-8, Paper 2003-01-1555, 22-28. SAE International, Troy, MI, USA.

36. Ghanem R, Sarkar A (2003) Reduced models for the medium-frequency dynamics of stochastic systems. J Acoust Soc Am 113(2):834-846. doi:http://dx.doi.org/10.1121/1.1538246

37. Soize C (2003) Uncertain dynamical systems in the medium-frequency range. J Eng Mech-ASCE 129(9):1017-1027. doi:http://dx.doi.org/10.1061/(ASCE)07339399(2003)129:9(1017) 
38. Capiez-Lernout E, Soize C (2008) Robust updating of uncertain damping models in structural dynamics for low- and medium-frequency ranges. Mech Syst Signal Pr 22(8):1774-1792. doi:http://dx.doi.org/10.1016/j.ymssp.2008.02.005

39. Kassem M, Soize C, Gagliardini L (2011) Structural partitioning of complex structures in the medium-frequency range. An application to an automotive vehicle. J Sound Vib 330(5):937-946. doi:http://dx.doi.org/10.1016/j.jsv.2010.09.008

40. Ohayon R, Soize C (2014) Advanced Computational Vibroacoustics - Reduced-Order Models and Uncertainty Quantification. Cambridge University Press, New York.

41. Durand JF, Soize C, Gagliardini L (2008) Structuralacoustic modeling of automotive vehicles in presence of uncertainties and experimental identification and validation. J Acoust Soc Am 124(3):1513-1525. doi:http://dx.doi.org/10.1121/1.2953316

42. Arnoux A, Batou A, Soize C, Gagliardini L (2013) Stochastic reduced order computational model of structures having numerous local elastic modes in low frequency dynamics. J Sound Vib 332(16): 3667-3680. doi:http://dx.doi.org/10.1016/j.jsv.2013.02.019

43. Gagliardini L (2014) Dispersed vibroacoustic responses of industrial products: what are we able to predict? In: Proceedings of the International Conference on Noise and Vibration Engineering ISMA 2014, Leuven, Belgium, September 15-17, 17-37.

44. Bucher I, and Braun SG (1997) Left Eigenvectors: Extraction From Measurements and Physical Interpretation. J Appl Mech-T ASME 64(1):97-105. doi:http://dx.doi.org/10.1115/1.2787300

45. Hansen PC (1987) The Truncated SVD as a Method for Regularization. BIT 27(4):534-553. doi:http://dx.doi.org/10.1007/BF01937276

46. Guyan RJ (1965) Reduction of Stiffness and Mass Matrices. AIAA J 3(2):380-380. doi:http://dx.doi.org/10.2514/3.2874

47. Bouhaddi N, Fillod R (1992) A method for selecting master DOF in dynamic substructuring using the Guyan condensation method. Comput Struct 45(5-6):941-946. doi:http://dx.doi.org/10.1016/0045-7949(92)90052-2

48. Belytschko T, Mindle WL (1980) Flexural WavePropagation Behavior of Lumped Mass Approximations. Comput. Struct. 12(6):805-812. doi:http://dx.doi.org/10.1016/0045-7949(80)90017-6

49. Chan HC, Cai, CW, Cheung YK (1993) Convergence studies of dynamic analysis by using the finite element method with lumped mass matrix. J Sound Vib 165(2):193-207. doi:http://dx.doi.org/10.1006/jsvi.1993.1253

50. Jensen MS (1996) High convergence order finite elements with lumped mass matrix. Int J Numer Meth Eng 39(11):18791888. doi:http://dx.doi.org/10.1002/(SICI)10970207(19960615)39:11;1879::AID-NME933¿3.0.CO;2-2

51. Hahn Y, Kikuchi N (2005) Identification of global modeshape from a few nodal eigenvectors using simple free-form deformation. Eng Comput 21(2):115-128. doi:http://dx.doi.org/10.1007/s00366-005-0314-x

52. Guyader JL (2009) Characterization and reduction of dynamic models of vibrating systems with high modal density. J Sound Vib 328(4-5):488-506. doi:http://dx.doi.org/10.1016/j.jsv.2009.08.012

53. Guyader JL (1990) Modal sampling method for the vibration study of systems of high modal density. J Acoust Soc Am 88(5):2269-2276. doi:http://dx.doi.org/10.1121/1.400069

54. Noor A, Anderson M, Greene W (1978) Continuum models for beam- and platelike- lattice structures. AIAA J 16(12):1219-1228. doi:http://dx.doi.org/10.2514/3.61036

55. Planchard J (1995) Vibrations of nuclear fuel assemblies: A simplified model. Nucl Eng Des 86(3):383-391. doi:http://dx.doi.org/10.1016/0029-5493(85)90303-6

56. Sigrits J, Broc D (2008) Dynamic analysis of a tube bundle with fluid-structure interaction modelling using a homogenisation method. Comput Method Appl M 197(9-12):1080-1099. doi:http://dx.doi.org/10.1016/j.cma.2007.10.010

57. Craig RR (1985) A review of time domain and frequency domain component mode synthesis method in combined experimental-analytical modeling of dynamic structural systems. ASME-AMD 67, D.R. Martinez and A.K. Miller, New York.

58. de Klerk D, Rixen DJ, Voormeeren SN (2008) General framework for dynamic substructuring: History, review, and classification of techniques. AIAA J 46:1169-1181. doi:http://dx.doi.org/10.2514/1.33274

59. Leung AYT (1993) Dynamic stiffness and substructures. Springer-Verlag, Berlin.

60. Ohayon R, Soize C, Sampio R (2014) Variational-based reduced-order model in dynamic substructuring of coupled structures through a dissipative physical interface: Recent advances. Arch Comput Method E 21(3):321-329. doi:http://dx.doi.org/10.1007/s11831-014-9107-y

61. Argyris JH, Kelsey S (1959) The analysis of fuselages of arbitrary cross-section and taper: A DSIR sponsored research program on the development and application of the matrix force method and the digital computer. Aircr Eng Aerosp Tec 31(3):62-74. doi:http://dx.doi.org/10.1108/eb033088

62. Przemieniecki JS (1963) Matrix structural analysis of substructures. AIAA J 1(1):138-147. doi:http://dx.doi.org/10.2514/3.1483

63. Irons B (1965) Structural eigenvalue problems - elimination of unwanted variables. AIAA J 3(5):961-962. doi:http://dx.doi.org/10.2514/3.3027

64. Hurty WC (1960) Vibrations of structural systems by component mode synthesis. J Eng Mech-ASCE 86(4):5170 .

65. Hurty WC (1965) Dynamic analysis of structural systems using component modes. AIAA J 3(4):678-685. doi:http://dx.doi.org/10.2514/3.2947

66. Craig RR, Bampton MCC (1968) Coupling of substructures for dynamic analyses. AIAA J 6(7):1313-1319. doi:http://dx.doi.org/10.2514/3.4741

67. Bathe KJ, Gracewski S (1981) On nonlinear dynamic analysis using substructuring and mode superposition. Comput Struct 13(5):699-707. doi:http://dx.doi.org/10.1016/0045-7949(81)90032-8

68. Farhat C, Geradin M (1994) On a component mode synthesis method and its application to incompatible substructures. Comput Struct 51(5):459-473. doi:http://dx.doi.org/10.1016/0045-7949(94)90053-1

69. Meirovitch L, Hale AL (1981) On the substructure synthesis method. AIAA J 19(7):940-947. doi:http://dx.doi.org/10.2514/3.51023

70. Meirovitch L, Kwak MK (1991) RayleighRitz based substructure synthesis for flexible multibody systems. AIAA J 29(10):1709-1719. doi:http://dx.doi.org/10.2514/3.10794

71. Voormeeren SN, van der Valk PL, Rixen DJ (2011) Generalized methodology for assembly and reduction of component models for dynamic substructuring. AIAA J 49(5):1010-1020. doi:http://dx.doi.org/10.2514/1.J050724

72. Benfield WA, Hruda RF (1971) Vibration analysis of structures by component mode substitution. AIAA J 9(7):1255-1261. doi:http://dx.doi.org/10.2514/3.49936 
73. Mac Neal R (1971) A hybrid method of component mode synthesis. Comput Struct 1(4):581-601. doi:http://dx.doi.org/10.1016/0045-7949(71)90031-9

74. Rubin S (1975) Improved component-mode representation for structural dynamic analysis. AIAA J 13(8):9951006. doi:http://dx.doi.org/10.2514/3.60497

75. Markovic D, Park KC, Ibrahimbegovic A (2007) Reduction of substructural interface degrees of freedom in flexibility-based component mode synthesis. Int J Numer Meth Eng 70(2):163-180. doi:http://dx.doi.org/10.1002/nme.1878

76. Ohayon R, Sampaio R, Soize C (1997) Dynamic substructuring of damped structures using singular value decomposition. J Appl Mech-T ASME 64(2):292-298. doi:http://dx.doi.org/10.1115/1.2787306

77. Park KC, Park YH (2004) Partitioned component mode synthesis via a flexibility approach. AIAA J 42(6):12361245. doi:http://dx.doi.org/10.2514/1.10423

78. Rixen DJ (2004) A dual Craig-Bampton method for dynamic substructuring. J Comput Appl Math 168(1-2):383-391. doi:http://dx.doi.org/10.1016/j.cam.2003.12.014

79. Beck JL, Katafygiotis LS (1998) Updating models and their uncertainties - I: Bayesian statistical framework. J Eng Mech-ASCE 124(4):455461. doi:http://dx.doi.org/10.1061/(ASCE)07339399(1998)124:4(455)

80. Ibrahim RA (1985) Parametric Random Vibration. John Wiley and Sons, New York.

81. Ghanem RG, Spanos PD (1991) Stochastic Finite Elements: A Spectral Approach. Springer-Verlag, New York. Revised edition, Dover Publications, New York, 2003.

82. Kennedy MC, O'Hagan A (2001) Bayesian calibration of computer models. J R Statist Soc B 63:425-464. doi:http://dx.doi.org/10.1111/1467-9868.00294

83. Soize C, Ghanem R (2004) Physical systems with random uncertainties: Chaos representation with arbitrary probability measure. SIAM J Sci Comput 26(2):395-410. doi:http://dx.doi.org/10.1137/S1064827503424505

84. Mace R, Worden W, Manson G (2005) Uncertainty in Structural Dynamics. Special issue of the Journal of Sound and Vibration 288(3):431-790.

85. Schuëller GI (2005) Computational methods in stochastic mechanics and reliability analysis. Special issue of Computer Methods in Applied Mechanics and Engineering 194(12-16):1251-1795

86. Schuëller GI (2005) Uncertainties in Structural Mechanics and Analysis - Computational Methods. Special issue of Computer and Structures 83(14):1031-1150.

87. Schuëller GI (2006) Developments in stochastic structural mechanics. Arch Appl Mech 75(10-12):755-773. doi:http://dx.doi.org/10.1007/s00419-006-0067-z

88. Bayarri MJ, Berger JO, Paulo R, Sacks J, Cafeo JA, Cavendish J, Lin CH, Tu J (2007) A framework for validation of computer models. Technometrics 49(2):138-154, published online 01 Jan 2012 http://dx.doi.org/10.1198/004017007000000092

89. Deodatis G, Spanos PD (2008) 5th International Conference on Computational Stochastic Mechanics. Special issue of the Probabilistic Engineering Mechanics 23(23):103-346

90. Schuëller GI, Pradlwarter HJ (2009) Uncertain linear systems in dynamics: Retrospective and recent developments by stochastic approaches. Eng Struct 31(11):2507-2517. doi:http://dx.doi.org/10.1016/j.engstruct.2009.07.005

91. Le Maitre OP, Knio OM (2010) Spectral methods for uncerainty quantification with applications to computational fluid dynamics. Springer, Heidelberg.
92. Arendt PD, Apley DW, Chen W, Lamb D, Gorsich D (2012) Improving identifiability in model calibration using multiple responses. J Mech Des 134(10):100909. doi:http://dx.doi.org/10.1115/1.4007573

93. Soize C (2013) Stochastic modeling of uncertainties in computational structural dynamics - Recent theoretical advances. J Sound Vib 332(10):2379-2395. doi:http://dx.doi.org/10.1016/j.jsv.2011.10.010

94. Ghanem R, Higdon D, Owhadi H (Eds) (2017) Handbook of Uncertainty Quantification, Springer International Publishing Switzerland. doi:http://dx.doi.org/10.1007/978-3-319-11259-6

95. Bui-Thanh T, Willcox K, Ghattas O (2008) Parametric reduced-order models for probabilistic analysis of unsteady aerodynamic applications. AIAA J 46(10):25202529. doi:http://dx.doi.org/10.2514/1.35850

96. Degroote J, Virendeels J, Willcox K (2010) Interpolation among reduced-order matrices to obtain parameterized models for design, optimization and probabilistic analysis. Int J Numer Meth Fl 63(2):207-230. doi:http://dx.doi.org/10.1002/fld.2089

97. Marzouk YM, Najm HN, Rahn LA (2007) Stochastic spectral methods for efficient Bayesian solution of inverse problems. J Comput Phys 224(2):560-586. doi:http://dx.doi.org/10.1016/j.jcp.2006.10.010

98. Galbally D, Fidkowski K, Willcox K, Ghattas O (2010) Non-linear model reduction for uncertainty quantification in large scale inverse problems. Int J Numer Meth Eng 81(12):1581-1608. doi:http://dx.doi.org/10.1002/nme.2746

99. Lieberman C, Willcox K, Ghattas O (2010) Parameter and state model reduction for large scale statistical inverse problems. SIAM J Sci Comput 32(5):2523-2542. doi:http://dx.doi.org/10.1137/090775622

100. Nouy A, Soize C (2014) Random fields representations for stochastic elliptic boundary value problems and statistical inverse problems. Eur J Appl Math 25(3):339 373. doi:http://dx.doi.org/10.1017/S0956792514000072

101. Cui T, Marzouk YM, Willcox KE (2015) Data-driven model reduction for the Bayesian solution of inverse problems. Int J Numer Meth Eng 102(5):966-990. doi:http://dx.doi.org/10.1002/nme.4748

102. Soize C (2016) Random vectors and random fields in high dimension. Parametric model-based representation, identification from data, and inverse problems. pp:1-65, in Handbook for Uncertainty Quantification edited by Ghanem R, Higdon D, Owhadi H., Springer: Heidelberg. doi:http://dx.doi.org/10.1007/978-3-319-11259-6_30-1

103. Soize C (2000) A nonparametric model of random uncertainties for reduced matrix models in structural dynamics. Probabilist Eng Mech 15(3):277-294. doi:http://dx.doi.org/10.1016/S0266-8920(99)00028-4

104. Shannon CE (1948) A Mathematical Theory of Communication. Bell Syst Tech J 27(3), 379-423. doi:http://dx.doi.org/10.1002/j.15387305.1948.tb01338.x

105. Jaynes ET (1957) Information Theory and Statistical Mechanics. Phys. Rev. 106(4):620-630. doi:http://dx.doi.org/10.1103/PhysRev.106.620

106. Mignolet MP, Soize C (2008) Nonparametric stochastic modeling of linear systems with prescribed variance of several natural frequencies. Probabilist Eng Mech 23(2-3):267-278. doi:http://dx.doi.org/10.1016/j.probengmech.2007.12.027

107. Soize C (2016) C. Soize, Random matrix models and nonparametric method for uncertainty quantification, pp. 1-69, in Handbook of Uncertainty Quantification, edited by R. Ghanem, D. Higdon, and H. Owhadi, doi:http://dx.doi.org/10.1007/978-3-31911259-6_5-1, Springer International Publishing Switzer- 
land, 2016.

108. Chen C, Duhamel D, Soize C (2006) Probabilistic approach for model and data uncertainties and its experimental identification in structural dynamics: Case of composite sandwich panels. J Sound Vib 294(1-2):64-81. doi:http://dx.doi.org/10.1016/j.jsv.2005.10.013

109. Capillon R, Desceliers C, Soize C (2016) Uncertainty quantification in computational linear structural dynamics for viscoelastic composite structures. Comput Method Appl M 305:154-172. doi:http://dx.doi.org/10.1016/j.cma.2016.03.012

110. Soize C, Chebli H (2003) Random uncertainties model in dynamic substructuring using a nonparametric probabilistic model. J Eng Mech-ASCE 129(4):449457. doi:http://dx.doi.org/10.1061/(ASCE)07339399(2003)129:4(449)

111. Mignolet MP, Soize C, Avalos J (2013) Nonparametric stochastic modeling of structures with uncertain boundary conditions / coupling between substructures. AIAA J 51(6):1296-1308. doi:http://dx.doi.org/10.2514/1.J051555

112. Capiez-Lernout E, Soize C (2008) Robust design optimization in computational mechanics. J Appl Mech-T ASME 75(2):1-11. doi:http://dx.doi.org/10.1115/1.2775493

113. Mignolet MP, Soize C (2008) Stochastic reduced order models for uncertain geometrically nonlinear dynamical systems. Comput Method Appl M 197(45-48):3951-3963. doi:http://dx.doi.org/10.1016/j.cma.2008.03.032

114. Capiez-Lernout E, Soize C, Mignolet MP (2014) Post-buckling nonlinear static and dynamical analyses of uncertain cylindrical shells and experimental validation. Comput Method Appl M 271(1):210-230. doi:http://dx.doi.org/10.1016/j.cma.2013.12.011

115. Soize C, Farhat C (2016) Uncertainty quantification of modeling errors for nonlinear reduced-order computational models using a nonparametric probabilistic approach. Int J Numer Meth Eng, accepted 30 May 2016, first published 30 June 2016. doi:http://dx.doi.org/10.1002/nme.5312

116. Soize C, Batou A (2011) Stochastic reduced-order model in low-frequency dynamics in presence of numerous local elastic modes. J Appl Mech-T ASME 78(6):061003-1-9. doi:10.1115/1.4002593

117. Sethian JA (1995) A fast marching level set method for monotonically advancing fronts. P Natl Acad Sci USA 93(4):1591-1595.

118. Sethian JA, Kimmel R. Computing Geodesic Paths on Manifolds, P Natl Acad Sci USA 95: 8431-8435.

119. Karypis G, Vipin K (1998) A fast and high quality multilevel scheme for partitioning irregular graphs. SIAM J Sci Comput 20(1):359-392. doi:10.1137/S1064827595287997

120. Bennighof JK, Lehoucq RB (2004) An automated multilevel substructuring method for eigenspace computation in linear elastodynamics. SIAM J Sci Comput 25(6):2084-2016. doi:10.1137/S1064827502400650

121. Gao W, Li XS, Yang C, Bai Z (2008) An implementation and evaluation of the AMLS method for sparse eigenvalue problems. ACM T Math Software 34(4):20:120:28. doi:10.1145/1377596.1377600

122. Rubinstein R (1981) Simulation and the Monte Carlo Method. John Wiley and Sons, New York.

123. Golub GH, Van Loan CF (1983) Matrix Computations. John Hopkins Univ Press, Baltimore.

124. Inman HF, Bradley EL (1989) The overlapping coefficient as a measure of agreement between probability distributions and point estimation of the overlap of two normal densities. Commun Stat Theory 18(10): 3851-3874. doi:http://dx.doi.org/10.1080/03610928908830127 